\title{
MIMO Systems with Limited Rate Differential Feedback in Slowly Varying Channels
}

\author{
Taejoon Kim, Student Member, IEEE, David J. Love, Senior Member, IEEE, and Bruno Clerckx, Member, IEEE,
}

\begin{abstract}
In this paper, an adaptive limited feedback linear precoding technique for temporally correlated multiple-input multiple-output (MIMO) channels is proposed, where the receiver has perfect channel knowledge but the transmitter only receives a quantized channel direction. To perform adaptation to the time correlation structure, we employ a differential feedback, where the "amount" of the perturbation added to the previous precoder is determined by the statistics of the directional variation. Based on random matrix quantization analysis, we develop a spherical cap codebook approach, where the cap is centered at the previous precoder and the radius of the cap is determined proportional to the identified directional variation. If the channel is highly correlated in time, the proposed differential feedback scheme can achieve a throughput improvement in the large codebook size regime. The rest of the paper is devoted to developing a systematic spherical cap codebook generation method. The developed approach employs a feedback scheme that uses a differential rotation of the previously used precoder. Our codebook adaptation is based on generating a perturbation in Euclidean space and projecting the perturbation onto the unitary space. Simulation results show that the proposed adaptation scheme accurately tracks the channel using only a small rate of feedback.
\end{abstract}

Index Terms-Multiple-input multiple-output (MIMO) channel, adaptive linear precoding, limited feedback, differential feedback, temporal correlation.

\section{INTRODUCTION}

$\mathbf{M}$ ULTIPLE-input multiple-output (MIMO) signaling schemes allowing the transmitter to adapt to the channel state information (CSI) (often called closed-loop MIMO) have become a promising technology to support the increased demand for data rates expected in the coming years. While the receiver can obtain CSI using techniques such as training, the transmitter often requires CSI to be sent as feedback on the reverse link. In reality, perfect CSI is always unrealistic in practical communication systems. This fact motivates research on limited feedback. Recently, limited feedback MIMO systems have evolved into a key technique for the next generation and beyond (i.e., $4 \mathrm{G}$ and beyond) broadband wireless standards (see the references in [1]).

Limited feedback frameworks have been proposed for precoded spatial multiplexing systems (e.g., [2]-[4]). The pro-

T. Kim and D. J. Love are with the School of Electrical and Computer Engineering, Purdue University, West Lafayette, IN, 47906 USA (e-mail: kim487@ecn.purdue.edu,djlove@ecn.purdue.edu).

B. Clerckx is with Samsung Advanced Institute of Technology, Samsung Electronics, Yongin-Si, Gyeonggi-Do, 446-712 Korea (e-mail: bruno.clerckx@samsung.com).

This work was supported in part by Samsung Electronics and by Doctoral Fellowship Program funded by the Korean government (MOCIE). The materials in this paper have been presented in part at the IEEE Globecom conference, New Orleans, LA, December, 2008. posed works deal with how to quantize and feed back some kind of information about the multidimensional channel. The works in [2]-[4] provide common insight that quantizing the channel direction requires fewer degrees of freedom and provides more robust performance than directly quantizing the channel. In these systems, the transmit precoder is chosen from a finite set of precoding matrices, called the codebook, known to both the receiver and the transmitter, and the chosen codeword index is sent back from the receiver to the transmitter. In these works, an independent block-by-block fading channel is assumed.

Unlike the independent block fading channel, a temporally correlated channel models the current channel realization as dependent on the previous channel realizations and more closely models the real channel. In [5], an extensive capacity analysis is performed for a temporally correlated channel modeled by a first-order Gauss-Markov process. It is shown that even without CSI, multiple antenna systems utilizing the presence of temporal correlation provide performance benefits [5]. If the codebook is changed to match with some local statistics (either in time, space, or frequency), the codebook is called an adaptive codebook [6]. The channel subspace tracking problem has been investigated in the context of using an adaptive codebook [7]-[15]. The channel subspace tracking algorithm using the minimal amount of feedback (i.e., 1 bit of feedback) has been proposed by employing a stochastic perturbation approach [7]. The stochastic perturbation idea in [7] has been extended to [8] where the trajectory of the channel subspace variation is modeled by a geodesic on the Grassmannian manifold. Imposing a structural constraint to CSI reduces the degrees of freedom needed for representing $\mathrm{CSI}$, and using a joint Gaussian vector quantization (VQ) gives a further performance benefit [8]. In [9], the channel has been modeled by a first-order Gauss-Markov process and a temporal codebook switching via a supercodeset has been proposed. A spherical cap-based codebook switching scheme, where the spherical cap codebook is selected in a supercodeset containing various spherical cap codebooks with different centers and radii, has been proposed in [10]. Progressive refinements of beamforming vectors using spherical cap codebook structure have been studied in [11]. The drawback of methods in [9] and [10] comes from the fact that they require additional periodic feedback to inform the transmitter of the codebook index in the supercodeset. The period of this additional feedback depends on the amount of temporal correlation. Codebook switching in a supercodeset is also a focus in [12], [13]. However, [12], [13] does not need the additional feedback for the codebook index because the codebook switching follows a 
predefined mechanism based on the state transition statistics. An online adaptive codebook scheme that does not require codebook switching has been considered by employing the rotation of the previous precoder in [14]. Two different codebooks are employed for tracking low speed channels and high speed channels in [15]. Other work has investigated adaptive feedback designs in [12], [16]-[18]. Although these are not adaptive codebook approaches, these works show benefits by using techniques such as variable length feedback encoding [12], [13], carefully controlled feedback rate and/or update period [16], [17], and differential channel quality indicator (CQI) feedback [18].

Closed-loop MIMO can achieve higher rates than open-loop MIMO because it optimizes the transmit covariance matrix as a function of the channel conditions. When the transmitter has perfect channel knowledge, the capacity is achieved by waterfilling [19]. When the feedback rate is limited, to realize quantized waterfilling, both the quantized channel subspace and quantized power allocation can be sent back [20] or feedback of the quantized covariance matrix can be employed [21], [22]. Note that the codebooks in [20]-[22] utilize a waterfilling-based transmit covariance matrix design, whereas the works in [2]-[4], [7]-[10], [12], [14], [15] allocate equal power along each stream and focus on subspace codebook design. These two techniques are different. The former is called explicit feedback and the later is called implicit feedback. On top of the technical difference, practical systems such as IEEE $802.16 m$ [23] and 3GPP LTE [24] focus on implicit feedback. In the context of explicit feedback, differential covariance matrix feedback in temporally correlated channel is proposed in [25], [26] where the trajectory of the channel covariance matrix is modeled by a geodesic in a positive definite matrix space [27]. Though comparing the proposed scheme to an explicit feedback scheme [25] is not a fair comparison, we provide simulations in Section V to emphasize the efficiency of our scheme.

In this paper, we develop an adaptive codebook scheme for MIMO spatial multiplexing systems operating in temporally correlated channels. A spherical cap codebook-based adaptation scheme is proposed. The adaptation to the time correlation is performed by controlling the spherical cap radius, where the radius is determined by integrating the amount of the channel directional variation and the amount of the quantization error propagated from the previous quantization stages. The key analytical framework used to quantify the temporal statistic is random matrix quantization. The spherical cap radius is progressively updated and refined to cope with quantization error accumulation inherent to differential feedback approaches. Next, we propose a systematic method to generate and update the spherical cap codebook. To facilitate the systematic generation, differential rotation of the previous precoder is employed, where the amount of the rotation (or perturbation) applied to the previous precoder is determined by the spherical cap radius. For this set-up, we first consider a general rotation codebook design problem. Then, this general rotation codebook is extended to produce the perturbation set and the spherical cap codebook is designed by projecting the perturbations onto the precoder space. We propose two ap- proaches using the two respective perturbation set generation methods.

The basic idea of the perturbation and projection-based adaptation is not new. This approach is popular in subspace estimation and tracking problems with unitary constraints [28]-[31]. The gradient assisted cost function $\mathcal{J}(\mathbf{w})$ (with ${ }^{1} \mathbf{w} \in \mathcal{U}(m, 1)$ ) maximization (or minimization) problem is surveyed and investigated in [28]. For instance, using ideas from [28], the vector at time index $n-1, \mathbf{w}(n-1) \in \mathcal{U}(m, 1)$ could be perturbed according to $\overline{\mathbf{w}}(n)=\mathbf{w}(n-1)+\mathbf{g}(n-1)$ in Euclidean space $\mathbb{C}^{m \times 1}$ and projected as $\mathbf{w}(n)=\frac{\overline{\mathbf{w}}(n)}{\|\overline{\mathbf{w}}(n)\|}$. The function $\mathrm{g}(n-1)$ denotes the gradient vector defined by $\mathbf{g}(n-1)=\mu(n-1) \frac{\partial \mathcal{J}(\mathbf{w}(n-1))}{\partial \mathbf{w}(n-1)}$ where $\mu(n-1)$ denotes the step size to be designed. This gradient-based perturbation and orthonormal projection approach is addressed for a matrix case where the projection is performed based on Procrustes orthonormalization [29] or Gram-Schmidt orthonormalization [30]. The specific applications of [28]-[30] would be the feedback assisted stochastic gradient approach [7] and tangent space perturbed geodesic approach [8]. Though presented in a different context in [31], a subspace interpolation (or estimation) problem is investigated by perturbing the observation vectors in Euclidean space and projecting them to unitary space. The technique in [31] is extended to spherical linear interpolation [32] and unitary matrix linear interpolation [33] techniques for estimating the beamformer and precoder in the frequency domain. In [7], [8], [32], [33], an orthonormally projected perturbation codebook is generated at each channel use and the receiver chooses the best precoder via precoder selection rules, and the particular index is conveyed to the transmitter. Both the transmitter and receiver share the common perturbation and projection strategy. Schemes in [7], [8], [32], [33] use similar approaches to generate projected perturbation set with slight modifications for example, adding weighted average [32], [33], perturbing the tangent space [7], [8], applying rotation [33], and using different projection strategies.

Our adaptive codebook method is related to those methods in [7]-[10], [12]. However, different from [7]-[10], [12], adaptation to the channel statistic is based on the analysis of the quantization error and parametrization of the channel evolution statistic. Similar to [7], [8], the algorithm is based on generating a perturbation set and projecting the set's members onto the unitary space. The method of generating the perturbation is not restricted to only the tangent space in our approach. In addition, compared to [7], [8], our approach successively refines the amount of the perturbation and the rotation codebook does not depend on the number of transmission streams. Compared to [10], our approach does not

\footnotetext{
${ }^{1} \mathcal{U}(m, n)$ denotes the set of $m \times n$ matrices with orthonormal columns, $\mathbf{I}_{M}$ denotes the $M \times M$ identity matrix, $\mathbf{0}_{m \times n}$ denotes the $m \times n$ zero matrix, $T$ denotes transposition, ${ }^{*}$ denotes conjugate transposition, a bold capital letter A denotes the matrix, a bold lowercase letter a denotes the vector, $\operatorname{diag}\left(a_{1}, \ldots, a_{m}\right)$ denotes a square diagonal matrix with $a_{1}, \ldots, a_{m}$ along the diagonal, $\|\mathbf{A}\|_{F}$ denote the matrix Frobenious norm, $\|\mathbf{a}\|$ denotes the vector 2-norm, $\lambda_{k}(\mathbf{A})$ denotes the $k$ th largest singular value of $\mathbf{A}, \operatorname{tr}(\mathbf{A})$ denotes the matrix trace, $\operatorname{det}(\mathbf{A})$ denotes the matrix determinant, $\operatorname{vec}(\mathbf{A})$ reshapes $\mathbf{A} \in \mathbb{C}^{m \times n}$ into a $m n \times 1$ vector by stacking $\mathbf{A}$ columnwise, and $\Gamma(\cdot)$ denotes Gamma function.
} 
need to design a supercodeset and does not require additional codebook indicator feedback. The radius of the spherical cap follows statistics of the directional variation.

The remainder of this paper is organized as follows. In Section II, our system model and problem statement are presented. In Section III, statistics of the channel directional variation in the temporally correlated channel are quantified and throughput analysis is performed. In Section IV, a general rotation codebook design problem is investigated and a systematic rotation-based differential feedback framework is proposed. Simulation results and related discussions are given in Section V, and we close by providing conclusions in Section VI.

\section{SYSTEM OVERVIEW}

The channel is modeled as a stochastic process. Then, a general approach to differential feedback is introduced, and an extension of this scheme to rotation-based differential feedback is addressed.

\section{A. System Model}

A limited feedback MIMO spatial multiplexing system employing precoding with $M_{t}$ transmit antennas and $M_{r}$ receive antennas is considered. The transmit symbol vector at the channel instance $m$ (for $m=0,1, \ldots$ ) is denoted by $\mathbf{s}_{m}=\left[\begin{array}{lll}s_{m, 1} & \cdots s_{m, M}\end{array}\right]^{T} \in \mathbb{C}^{M \times 1}$ with $\mathbf{s}_{m} \sim \mathcal{C N}\left(\mathbf{0}_{M \times 1}, \mathbf{I}_{M}\right)$ and $M \leq \min \left\{M_{t}, M_{r}\right\}$. The vector $\mathbf{F}_{m} \mathbf{s}_{m}$ is sent through the channel where $\mathbf{F}_{m}$ denotes a precoding matrix. Then, the received signal is represented by

$$
\mathbf{y}_{m}=\sqrt{\frac{\rho}{M}} \mathbf{H}_{m} \mathbf{F}_{m} \mathbf{s}_{m}+\mathbf{n}_{m}
$$

where $\mathbf{n}_{m} \in \mathbb{C}^{M_{r} \times 1}$ denotes the noise vector with $\mathbf{n}_{m} \sim$ $\mathcal{C N}\left(\mathbf{0}_{M_{r} \times 1}, \mathbf{I}_{M_{r}}\right)$ and $\rho$ denotes the SNR. The matrix $\mathbf{H}_{m} \in$ $\mathbb{C}^{M_{r} \times M_{t}}$ represents a spatially uncorrelated Rayleigh flat fading channel matrix, whose entries are i.i.d. according to $\mathcal{C N}(0,1)$.

The evolution of $\mathbf{H}_{m}$ is modeled by a first-order GaussMarkov process

$$
\mathbf{H}_{m}=\epsilon \mathbf{H}_{m-1}+\sqrt{1-\epsilon^{2}} \mathbf{N}_{m}
$$

where $\mathbf{N}_{m} \in \mathbb{C}^{M_{r} \times M_{t}}$ has i.i.d. entries with distribution $\sim \mathcal{C N}(0,1)$ and $E\left[\operatorname{vec}\left(\mathbf{H}_{m-1}\right)^{*} \operatorname{vec}\left(\mathbf{N}_{m}\right)\right]=\mathbf{0}_{M_{r} M_{t} \times M_{r} M_{t}}$. The noise process $\mathbf{n}_{m}$ in (1) is independent of $\mathbf{N}_{m}$ and $\mathbf{H}_{0}$. The time correlation coefficient $\epsilon(0 \leq \epsilon \leq 1)$ represents the correlation between elements $h_{m, i, j}$ and $h_{m-1, i, j}$ (where $h_{m, i, j}$ denotes the $(i, j)$ entry of $\left.\mathbf{H}_{m}\right)$. We assume all the elements of $\mathbf{H}_{m}$ have the same $\epsilon$. The evolution variable $\epsilon$ obeys Jakes' model [34] according to $\epsilon=J_{0}\left(2 \pi f_{D} T\right)$, where $J_{0}(\cdot)$ is the zeroth order Bessel function, $T$ denotes the channel instantiation interval, and $f_{D}=\frac{v f_{c}}{c}$ denotes the maximum Doppler frequency using terminal velocity $v$, carrier frequency $f_{c}$, and $c=3 \times 10^{8} \mathrm{~m} / \mathrm{s}$.

\section{B. Capacity Selection Criterion}

We assume that the receiver perfectly knows the current channel. Then, the instantaneous mutual information between $\mathbf{s}_{m}$ and $\mathbf{y}_{m}$ for a given channel $\mathbf{H}_{m}$ is known to be

$$
I\left(\mathbf{F}_{m}\right)=\log _{2}\left(\operatorname{det}\left(\mathbf{I}_{M}+\frac{\rho}{M} \mathbf{F}_{m}^{*} \mathbf{H}_{m}^{*} \mathbf{H}_{m} \mathbf{F}_{m}\right)\right) .
$$

We focus on equal power allocation (i.e., $\mathbf{F}_{m} \in \mathcal{U}\left(M_{t}, M\right)$ ). Using precoder $\mathbf{F}_{m}$ instead of waterfilling with covariance feedback typically results in only a small rate degradation and provides numerous practical benefits [35]. Equal power allocation can also be combined with multimode (or rank) adaptation [4], [36], and rank adapted equal power allocation precoding has been adopted in current standards [23], [24].

Denote the precoding codebook $\mathcal{F}_{m}=\left\{\mathbf{F}_{m, i}\right\}_{i=1}^{K}$ with $K=$ $2^{B}$ and $\mathbf{F}_{m, i} \in \mathcal{U}\left(M_{t}, M\right)$. The subscript $m$ is used to indicate that $\mathcal{F}_{m}$ varies with the channel index $m$. Fig. 1 illustrates the operation of the proposed limited feedback precoding system. As shown in Fig. 1, the codebook is updated at each channel instance. Given the quantized precoder, both transmitter and receiver share the same codebook update scheme which will be addressed in Section II-C. At the receiver side, the precoder $\mathbf{F}_{m} \in \mathcal{F}_{m}$ is chosen according to the capacity criterion [2]-[4]

$$
\mathbf{F}_{m}=\underset{\mathbf{F}_{m, i} \in \mathcal{F}_{m}}{\operatorname{argmax}} I\left(\mathbf{F}_{m, i}\right)
$$

Note that (4) is also equivalent to minimizing the determinant of the mean squared error (MSE) matrix.

The singular value decomposition (SVD) of the channel is $\mathbf{H}_{m}=\mathbf{U}_{m} \boldsymbol{\Sigma}_{m} \mathbf{V}_{m}^{*}$, where $\mathbf{U}_{m} \in \mathcal{U}\left(M_{r}, M_{r}\right), \mathbf{V}_{m} \in$ $\mathcal{U}\left(M_{t}, M_{t}\right)$, and $\boldsymbol{\Sigma}_{m} \in \mathbb{R}^{M_{r} \times M_{t}}$ is a singular value matrix with $\lambda_{k}\left(\mathbf{H}_{m}\right)$ at position $(k, k)$ for $k=1, \ldots, \min \left\{M_{t}, M_{r}\right\}$. If we denote a matrix formed by taking the first $M$ columns of $\mathbf{V}_{m}$ as $\overline{\mathbf{V}}_{m}$, the $\overline{\mathbf{V}}_{m}$ is the optimal unitary precoder that maximizes the effective channel power, thereby maximizing the mutual information [2]. In addition, throughout the paper, in order to measure the principal angle differences between subspaces $\mathbf{S}_{1} \in \mathcal{U}\left(M_{t}, M\right)$ and $\mathbf{S}_{2} \in \mathcal{U}\left(M_{t}, M\right)$, the chordal distance defined for the Grassmann manifold is given by

$$
d_{c}\left(\mathbf{S}_{1}, \mathbf{S}_{2}\right)=\sqrt{M-\left\|\mathbf{S}_{1}^{*} \mathbf{S}_{2}\right\|_{F}^{2}} .
$$

In what follows, we show that the precoder selection criterion in (4) is related to the direction mismatch between $\overline{\mathbf{V}}_{m}$ and $\mathbf{F}_{m}$ measured by the chordal distance between the precoder subspaces.

Define the average minimum achievable rate loss (or distortion) as

$$
D\left(\mathcal{F}_{m}\right)=E\left[\left(I\left(\overline{\mathbf{V}}_{m}\right)-I\left(\mathbf{F}_{m}\right)\right)\right]
$$

where $I\left(\overline{\mathbf{V}}_{m}\right)=\log _{2}\left(\operatorname{det}\left(\mathbf{I}_{M}+\frac{\rho}{M} \overline{\boldsymbol{\Sigma}}_{m}^{2}\right)\right)$. Here $\overline{\boldsymbol{\Sigma}}_{m} \in$ $\mathbb{C}^{M \times M}$ is the diagonal matrix formed by taking the first $M$ rows and columns of $\boldsymbol{\Sigma}_{m}$. Then, $D\left(\mathcal{F}_{m}\right)$ can be upper bounded 


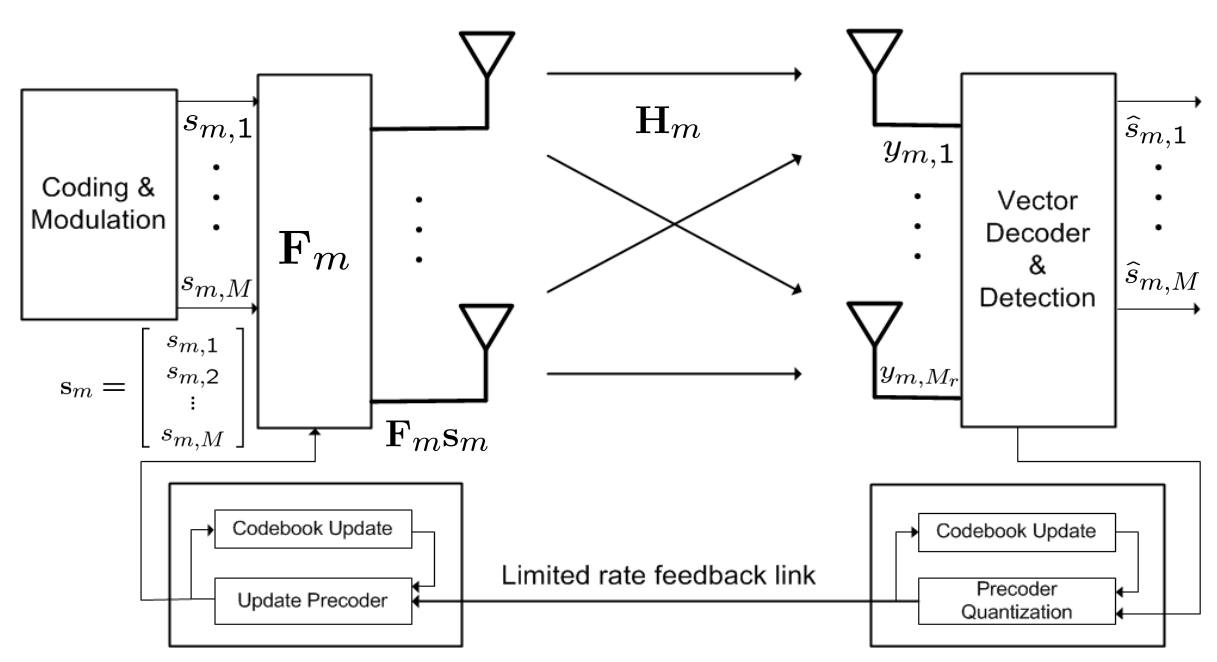

Fig. 1. Block diagram of the proposed MIMO spatial multiplexing system with limited rate differential feedback.

by

$$
\begin{aligned}
& D\left(\mathcal{F}_{m}\right) \leq E\left[\log _{2}\left(\frac{\operatorname{det}\left(\mathbf{I}_{M}+\frac{\rho}{M} \overline{\boldsymbol{\Sigma}}_{m}^{2}\right)}{\operatorname{det}\left(\mathbf{I}_{M}+\frac{\rho}{M} \mathbf{F}_{m}^{*} \overline{\mathbf{V}}_{m} \overline{\mathbf{\Sigma}}_{m}^{2} \overline{\mathbf{V}}_{m}^{*} \mathbf{F}_{m}\right)}\right)\right] \\
&=E\left[\operatorname{tr}\left(\log _{2}\left(\mathbf{I}_{M}+\frac{\rho}{M} \overline{\boldsymbol{\Sigma}}_{m}^{2}\right)\right)\right. \\
&\left.\quad-\operatorname{tr}\left(\log _{2}\left(\mathbf{I}_{M}+\frac{\rho}{M} \mathbf{F}_{m}^{*} \overline{\mathbf{V}}_{m} \overline{\mathbf{\Sigma}}_{m}^{2} \overline{\mathbf{V}}_{m}^{*} \mathbf{F}_{m}\right)\right)\right] \\
& \leq E\left[\frac{1}{\ln (2)} \frac{\rho}{M} \operatorname{tr}\left(\overline{\boldsymbol{\Sigma}}_{m}^{2}\left(\mathbf{I}_{M}-\overline{\mathbf{V}}_{m}^{*} \mathbf{F}_{m} \mathbf{F}_{m}^{*} \overline{\mathbf{V}}_{m}\right)\right)\right]
\end{aligned}
$$

where $\mathbf{G}_{i} \in\left\{\mathbf{G}_{i}\right\}_{i=1}^{2^{B}}$ (with $\mathbf{G}_{i} \in \mathbb{C}^{M \times M}$ ) denotes the Gaussian codeword used to perturb the tangent space of $\mathbf{F}_{m-1}$. By iterating (7) from $i=1$ to $2^{B}$, the function $g$ generates a size $2^{B}$ codebook $\mathcal{F}_{m}$ as the points on the geodesic lines defined on the Grassmannian manifold. The length of the arc is specified by the parameter $a \geq 0$ which impacts the performance of the algorithm. An improper choice of $a$ results in quantization error accumulation and fails to track $\overline{\mathbf{V}}_{m}$. In [8], $a$ is found by Monte-Carlo simulation so that it shows the best tracking performance.

Now, we describe a differential feedback approach based on random spherical cap codebook construction. We introduce an abstract function $\varsigma: \mathcal{U}\left(M_{t}, M\right) \rightarrow \mathcal{U}\left(M_{t}, M\right)$. Here, the function $\varsigma$ realizes a random matrix in a spherical cap centered at $\mathbf{F}_{m-1}$ with radius $r_{m}$. Specifically, given $\mathbf{F}_{m-1}$, a random codeword $\mathbf{F}$ is realized by

$$
\mathbf{F}=\varsigma\left(\mathbf{F}_{m-1}, r_{m}\right)
$$

Using $2^{B}$ random realizations from the function in (8), we create a random spherical cap codebook $\mathcal{F}_{m}$. This codebook will be used for analytical purposes to allow the characterization of the directional variation from $\mathbf{F}_{m-1}$ to $\overline{\mathbf{V}}_{m}$. The statistic of the directional variation is measured by $r_{m}$ and related to the chordal metric. Given the initial codebook $\mathcal{F}_{0}$, the random codebook-based differential feedback operates recursively by applying the precoder selection (i.e., (4)) and codebook update (i.e., (8)).

Compared to (7), the function in (8) generates a perturbation point in a spherical cap and $r_{m}$ is successively refined as the channel evolves with $m$. The main focus of our paper is how to determine $r_{m}$ in (8) by integrating the effects of the channel directional variation and the accumulated quantization error. Section III is devoted to characterizing $r_{m}$. 


\section{Rotation-Based Differential Feedback Framework}

The codebook evolution in (8) is based on a random codebook construction. For the sake of application, a systematic codebook generation is of interest. We introduce a basic idea for a rotation-based differential feedback framework. Define a rotation-based codeword evolution function $\vartheta: \mathbb{C}^{M_{t} \times M_{t}} \times$ $\mathcal{U}\left(M_{t}, M\right) \rightarrow \mathcal{U}\left(M_{t}, M\right)$ and

$$
\mathbf{F}_{m, i}=\vartheta\left(\mathbf{F}_{m-1}, r_{m} \boldsymbol{\Theta}_{i}\right)
$$

where $\boldsymbol{\Theta}_{i} \in \mathcal{U}\left(M_{t}, M_{t}\right)$ is a rotation codeword in a rotation codebook $\mathcal{Q}=\left\{\boldsymbol{\Theta}_{i}\right\}_{i=1}^{2^{B}}$. Using this function $2^{B}$ times, we can create a spherical cap codebook $\mathcal{F}_{m}$ around $\mathbf{F}_{m-1}$ by rotating $\mathbf{F}_{m-1}$ using a rotation codeword $\boldsymbol{\Theta}_{i} \in \mathcal{Q}$ (i.e., $\boldsymbol{\Theta}_{i} \mathbf{F}_{m-1}$ ), where the amount of the rotation applied to $\mathbf{F}_{m-1}$ is proportional to $r_{m}$. Details of this codebook adaptation are discussion in Section IV.

\section{Differential Feedback ANd Performance ANALYSIS}

In this section, a bound on the average directional variation measured by chordal distance is characterized using the codebook evolution defined in (8). The codebook $\mathcal{F}_{m}$ is then generated as a random spherical cap codebook using the radius $r_{m}$. Throughput analysis of the proposed differential feedback scheme is also presented.

\section{A. Average Directional Variation}

Given the previous precoder $\mathbf{F}_{m-1}$, the spherical cap radius $r_{m}$ in (8) is characterized by measuring the average directional variation from $\mathbf{F}_{m-1}$ to $\overline{\mathbf{V}}_{m}$. From the channel evolution model in (2), directly characterizing this quantity is intractable. To measure this quantity in terms of chordal distance, consider the average effective channel power loss induced by $\mathbf{F}_{m-1}$

$$
E\left[\left\|\mathbf{H}_{m} \overline{\mathbf{V}}_{m}\right\|_{F}^{2}\right]-E\left[\left\|\mathbf{H}_{m} \mathbf{F}_{m-1}\right\|_{F}^{2}\right],
$$

where $\mathbf{H}_{m}$ follows (2) and the expectation is taken with respect to $\mathbf{H}_{m}$ and $\mathbf{F}_{m-1}$. The effective power leakage induced in (10) is related to the directional mismatch between $\mathbf{F}_{m-1}$ and $\overline{\mathbf{V}}_{m}$. We want to extract an expression for the average directional variation by factoring (10) into the channel amplitude and the channel directional components. Denote the singular value decomposition of $\mathbf{N}_{m}$ as $\mathbf{X}_{m} \boldsymbol{\Lambda}_{m} \mathbf{P}_{m}^{*}$ in (2), where $\mathbf{X}_{m} \in \mathcal{U}\left(M_{r}, M_{r}\right), \mathbf{P}_{m} \in \mathcal{U}\left(M_{t}, M_{t}\right)$, and $\boldsymbol{\Lambda}_{m} \in \mathbb{R}^{M_{r} \times M_{t}}$ is the singular value matrix of $\mathbf{N}_{m}$. Then, we obtain a bound

$$
E\left[\left\|\mathbf{H}_{m} \overline{\mathbf{V}}_{m}\right\|_{F}^{2}-\left\|\mathbf{H}_{m} \mathbf{F}_{m-1}\right\|_{F}^{2}\right] \leq E\left[\operatorname{tr}\left(\overline{\boldsymbol{\Sigma}}_{m}^{2}\right)\right] v_{m}
$$

where $v_{m}$ is given by

$$
v_{m}=\epsilon^{2} E\left[d_{c}^{2}\left(\mathbf{F}_{m-1}, \overline{\mathbf{V}}_{m-1}\right)\right]+\left(1-\epsilon^{2}\right) E\left[d_{c}^{2}\left(\overline{\mathbf{P}}_{m}, \mathbf{F}_{m-1}\right)\right] .
$$

Here $\overline{\mathbf{P}}_{m}$ is formed by taking the first $M$ columns of $\mathbf{P}_{m}$. The details of (11) are provided in Appendix (VII-A). Note that for $M_{r}=M=1$ (i.e., multiple-input single-output (MISO) beamforming case), the bound in (11) becomes equality.

We focus on the directional quantity $v_{m}$. The quantity $v_{m}$ characterizes the amount of average directional variation from $\mathbf{F}_{m-1}$ to $\overline{\mathbf{V}}_{m}$ expressed as the weighted sum of the average quantization error at $m-1$ (i.e., $\left.q_{m-1}=E\left[d_{c}^{2}\left(\mathbf{F}_{m-1}, \overline{\mathbf{V}}_{m-1}\right)\right]\right)$ and the average temporal variation at $m$ (i.e., $E\left[d_{c}^{2}\left(\overline{\mathbf{P}}_{m}, \mathbf{F}_{m-1}\right)\right]$ ). The use of chordal distance becomes apparent when we perform the quantization error analysis to quantify $q_{m-1}$ based on the random spherical cap codebook generation in (8). The analysis is possible by utilizing the spherical cap volume formula in [38].

In what follows, each of the terms in (12) will be quantified for $m=1,2, \ldots$. This will eventually result in a recursive formula for $r_{m}$.

\section{B. Recursion for Quantization Error and Spherical Cap Ra- dius}

The successive codebook evolutions in (8) and precoder quantization in (4) reveal that the differential feedback framework suffers from quantization error accumulation (or propagation). If $\mathbf{F}_{m-1}$ is improperly quantized, the quantization error induced in $\mathbf{F}_{m-1}$ propagates to the next quantization stage because of the dependency of $\mathcal{F}_{m}$ on $\mathbf{F}_{m-1}$. As a cure, we control $r_{m}$ in (8) to cope with the accumulation of the quantization error.

Now, we characterize each term in (12). The quantity $E\left[d_{c}^{2}\left(\overline{\mathbf{P}}_{m}, \mathbf{F}_{m-1}\right)\right]$ in (12) is characterized by

$$
E\left[d_{c}^{2}\left(\overline{\mathbf{P}}_{m}, \mathbf{F}_{m-1}\right)\right]=M-\sum_{i=1}^{M} E\left[\left\|\overline{\mathbf{P}}_{m}^{*} \mathbf{f}_{m-1, i}\right\|^{2}\right]=\frac{M\left(M_{t}-M\right)}{M_{t}},
$$

where $\mathbf{f}_{m-1, i}$ denotes the $i$ th column of $\mathbf{F}_{m-1}$. Since $\overline{\mathbf{P}}_{m}$ is isotropic in $\mathcal{U}\left(M_{t}, M\right)$ and is independent of $\mathbf{f}_{m-1, i}$, the quantity $\left\|\overline{\mathbf{P}}_{m}^{*} \mathbf{f}_{m-1, i}\right\|^{2}$ is beta distributed with mean $\frac{M}{M_{t}}$ and shape parameters $M$ and $M_{t}-M$.

A spherical cap (or metric ball) centered at $\mathbf{A}$ with radius $r$ is defined as

$$
\mathcal{S}_{\mathbf{A}}(r)=\left\{\mathbf{B}: d_{c}(\mathbf{A}, \mathbf{B}) \leq r, \mathbf{A} \in \mathcal{U}\left(M_{t}, M\right), \mathbf{B} \in \mathcal{U}\left(M_{t}, M\right)\right\} .
$$

In order to characterize the average quantization error, we characterize $q_{m}=E\left[d_{c}^{2}\left(\mathbf{F}_{m}, \overline{\mathbf{V}}_{m}\right)\right]$. Determining the closedform expression of $q_{m}$ becomes involed because we do not know the distribution of $d_{c}^{2}\left(\mathbf{F}_{m}, \overline{\mathbf{V}}_{m}\right)$ when $\mathbf{F}_{m}$ is correlated with $\mathbf{F}_{m-1}$ and $\overline{\mathbf{V}}_{m}$. For this reason, we characterize $q_{m}$ for $m \geq 0$ by focusing on the limiting behavior as $B$ grows large. Based on the asymptotic bound, a recursive formula for the spherical cap radius $r_{m}$ is derived. For the analytical purpose, random matrix quantization codebooks are realized by drawing each codeword independently from the isotropic distribution in $\mathcal{U}\left(M_{t}, M\right)$ for $\mathcal{F}_{0}$ and on $\mathcal{S}_{\mathbf{F}_{m-1}}\left(r_{m}\right)$ for $\mathcal{F}_{m}$ (with $m \geq 1$ ), respectively.

Lemma 1: For $1 \leq M \leq M_{t}-1, m=0,1, \ldots$, and $B$ sufficiently large, the average quantization error induced in the random spherical cap codebook $\mathcal{F}_{m}$ designed by (8) is bounded by

$$
q_{m} \leq \epsilon^{2 m} D_{0} 2^{-\frac{m B}{\kappa}}+\frac{\kappa\left(1-\epsilon^{2}\right)}{M_{t}}\left(\sum_{k=0}^{m-1} \epsilon^{2 k} 2^{-\frac{(k+1) B}{\kappa}}\right)+o(1),
$$

the average directional variation $v_{m+1}$ is upper bounded by

$$
v_{m+1} \leq \epsilon^{2(m+1)} D_{0} 2^{-\frac{m B}{\kappa}}+\frac{\kappa\left(1-\epsilon^{2}\right)}{M_{t}}\left(\sum_{k=0}^{m} \epsilon^{2 k} 2^{-\frac{k B}{\kappa}}\right)+o(1),
$$


and the squared radius $r_{m+1}^{2}$ is determined by taking the dominant term in (16) as

$$
r_{m+1}^{2}=\epsilon^{2(m+1)} D_{0} 2^{-\frac{m B}{\kappa}}+\frac{\kappa\left(1-\epsilon^{2}\right)}{M_{t}}\left(\sum_{k=0}^{m} \epsilon^{2 k} 2^{-\frac{k B}{\kappa}}\right)
$$

where $\kappa=M\left(M_{t}-M\right), D_{0}=\frac{1}{\kappa\left(C_{M_{t}, M}\right)^{\frac{1}{\kappa}}} \cdot \beta\left(C_{M_{t}, M} ; \frac{1}{\kappa}, 2^{B}+1\right)$, and $C_{M_{t}, M}=(\Gamma(\kappa+1))^{-1} \prod_{i=1}^{M} \frac{\Gamma\left(M_{t}-i+1\right)}{\Gamma(M-i+1)}$.

Proof: See Appendix VII-B.

If $\epsilon$ is known to both the transmitter and receiver, the transmitter and receiver can compute $r_{m}$ using (17). This can be accomplished by having the receiver measure $\epsilon$ and share this long term statistic with transmitter. Since $\epsilon$ is a long term statistic, the overhead to feed back $\epsilon$ is negligible compared to instantaneous $B$ bits feedback.

\section{Throughput Analysis}

By taking the temporal correlation into account in the feedback design, a lower distortion quantization is expected. To examine this, we investigate the achievable throughput performance of the random spherical cap-based differential feedback scheme.

For sufficiently large $B$, plugging (15) in (6) yields

$$
\begin{array}{r}
D\left(\mathcal{F}_{m}\right) \leq \frac{\rho}{\ln (2)} E\left[\lambda_{1}^{2}\right]\left[\epsilon^{2 m} D_{0} 2^{-\frac{m B}{\kappa}}+\left(1-\epsilon^{2}\right) \frac{\kappa}{M_{t}}\right. \\
\left.\times\left(\sum_{k=0}^{m-1} \epsilon^{2 k} 2^{-\frac{(k+1) B}{\kappa}}\right)\right]+o(1) .
\end{array}
$$

Now, the distortion can be further analyzed at both low and high SNR.

1) Low SNR: At low SNR, the optimal transmission strategy is to beamform (i.e., $M=1$ ) on the strongest eigenmode of the channel [19]. Then, with $M=1$ and at the steady state $(m \rightarrow \infty)$, (18) converges to

$$
\mathcal{D}\left(\mathcal{F}_{m}\right) \stackrel{m \rightarrow \infty}{\leq} \frac{\rho}{\ln (2)} E\left[\lambda_{1}^{2}\right]\left(\frac{M_{t}-1}{M_{t}}\right)\left(\frac{1-\epsilon^{2}}{2^{\frac{B}{M_{t}-1}}-\epsilon^{2}}\right)+o(1)
$$

Note that in the conventional feedback schemes (e.g., [2]-[4]), the codebook $\mathcal{F}_{0}$ is constantly used for $m \geq 0$. In this case, the average capacity distortion (6) with $M=1$ can be bounded by

$$
\begin{aligned}
\mathcal{D}\left(\mathcal{F}_{0}\right) & \leq \frac{\rho}{\ln (2)} E\left[\lambda_{1}^{2}\right] \frac{1}{M_{t}-1} \beta\left(\frac{1}{M_{t}-1}, 2^{B}+1\right) \\
& \leq \frac{\rho}{\ln (2)} E\left[\lambda_{1}^{2}\right] 2^{-\frac{B}{M_{t}-1}}
\end{aligned}
$$

where (20) follows from the fact that when $M=1, C_{M_{t}, M}$ in (44) becomes one, and the bound (44) holds as an equality. The bound in (21) follows from the bound $\frac{1}{M_{t}-1} \beta\left(\frac{1}{M_{t}-1}, 2^{B}+1\right) \leq$ $2^{-\frac{B}{M_{t^{-1}}}}$ in [39].

Comparing (19) and (21), in the large $B$ regime, significant throughput gain of the proposed scheme is possible when the channel is highly correlated $(\epsilon \approx 1)$, because the minor term $o(1)$ in (19) converges to zero faster than $2^{-\frac{B}{M_{t}-1}}$ as $B \rightarrow \infty$.
2) High SNR: In the high SNR regime, from (5),

$$
\begin{aligned}
\mathcal{D}\left(\mathcal{F}_{m}\right) & \stackrel{\rho \rightarrow \infty}{\lesssim} E\left[\log _{2}\left(\operatorname{det}\left(\frac{\rho}{M} \overline{\boldsymbol{\Sigma}}_{m}^{2}\right) / \operatorname{det}\left(\frac{\rho}{M} \mathbf{F}_{m}^{*} \overline{\mathbf{V}}_{m} \overline{\boldsymbol{\Sigma}}_{m}^{2} \overline{\mathbf{V}}_{m}^{*} \mathbf{F}_{m}\right)\right)\right] \\
& =E\left[\log _{2}\left(\operatorname{det}\left(\mathbf{F}_{m}^{*} \overline{\mathbf{V}}_{m} \overline{\mathbf{V}}_{m}^{*} \mathbf{F}_{m}\right)^{-1}\right)\right] \\
& \leq E\left[\left(M_{t}-1\right) \log _{2}\left(\frac{\operatorname{tr}\left(\left(\mathbf{F}_{m}^{*} \overline{\mathbf{V}}_{m} \overline{\mathbf{V}}_{m}^{*} \mathbf{F}_{m}\right)^{-1}\right)}{M_{t}-1}\right)\right]
\end{aligned}
$$

where the bound in (22) is due to the arithmetic-geometric mean inequality. At high SNR, the optimal transmit strategy is full spatial multiplexing (i.e., $M=M_{t}$ ) [19]. In our system, since the chordal metric is used, we restrict $M=M_{t}-1$. Denote the null space of $\overline{\mathbf{V}}_{m}$ as $\mathbf{v}_{m}^{\perp} \in \mathcal{U}\left(M_{t}, 1\right)$. Then, by defining $\mathbf{g}=\mathbf{F}_{m}^{*} \mathbf{v}_{m}^{\perp}$, we have $\mathbf{F}_{m}^{*} \overline{\mathbf{V}}_{m} \overline{\mathbf{V}}_{m}^{*} \mathbf{F}_{m}=\mathbf{I}_{M_{t}-1}-\mathbf{g g}^{*}$ and $\operatorname{tr}\left(\left(\mathbf{F}_{m}^{*} \overline{\mathbf{V}}_{m} \overline{\mathbf{V}}_{m}^{*} \mathbf{F}_{m}\right)^{-1}\right)$ in (22) can be rewritten

$$
\begin{aligned}
\operatorname{tr}\left(\left(\mathbf{F}_{m}^{*} \overline{\mathbf{V}}_{m} \overline{\mathbf{V}}_{m}^{*} \mathbf{F}_{m}\right)^{-1}\right) & =\operatorname{tr}\left(\mathbf{I}_{M_{t}-1}+\mathbf{g}\left(1-\mathbf{g}^{*} \mathbf{g}\right)^{-1} \mathbf{g}^{*}\right) \\
& =M_{t}-1+\frac{\|\mathbf{g}\|^{2}}{\left(1-\|\mathbf{g}\|^{2}\right)}
\end{aligned}
$$

where the first step is due to the matrix inversion lemma ${ }^{2}$. Realizing that $\|\mathbf{g}\|^{2}=d_{c}^{2}\left(\mathbf{F}_{m}, \overline{\mathbf{V}}_{m}\right)$, from (22) we have

$$
\begin{aligned}
\mathcal{D}\left(\mathcal{F}_{m}\right) & \leq E\left[\left(M_{t}-1\right) \log _{2}\left(1+\frac{1}{M_{t}-1} \cdot \frac{d_{c}^{2}\left(\mathbf{F}_{m}, \overline{\mathbf{V}}_{m}\right)}{1-d_{c}^{2}\left(\mathbf{F}_{m}, \overline{\mathbf{V}}_{m}\right)}\right)\right] \\
& \approx E\left[\left(M_{t}-1\right) \log _{2}\left(1+\frac{2 \cdot d_{c}^{2}\left(\mathbf{F}_{m}, \overline{\mathbf{V}}_{m}\right)}{M_{t}-1}\right)\right] \\
& \leq\left(M_{t}-1\right) \log _{2}\left(1+\frac{2}{M_{t}-1} \cdot E\left[d_{c}^{2}\left(\mathbf{F}_{m}, \overline{\mathbf{V}}_{m}\right)\right]\right)
\end{aligned}
$$

In (23), we used the expansion $\frac{x}{1-x}=x+x^{2}+x^{3}+\cdots$ for $0 \leq x<1$, took the first two terms $x+x^{2}$, and used the bound $x+x^{2} \leq 2 x$. The last step (24) is due to the Jensen's inequality. Then, as $m \rightarrow \infty$, the bound yields

$$
\mathcal{D}\left(\mathcal{F}_{m}\right) \stackrel{m \rightarrow \infty}{\lesssim}\left(M_{t}-1\right) \log _{2}\left(1+\left(\frac{2}{M_{t}}\right)\left(\frac{1-\epsilon^{2}}{2^{\frac{B}{\left(M_{t}-1\right)}}-\epsilon^{2}}\right)+o(1)\right) .
$$

In conventional feedback schemes, it is straightforward to show that (24) is replaced by

$$
\mathcal{D}\left(\mathcal{F}_{m}\right) \lesssim \log _{2}\left(1+\left(\frac{2}{M_{t}-1}\right)\left(2^{-\frac{B}{M_{t}-1}}\right)\right) .
$$

The bounds in (25) and (26) reveal that if there exists a rich time diversity (i.e., $\epsilon \approx 1$ ) and $B$ is large enough, we can still expect significant throughput gain from the proposed differential feedback scheme in the high SNR regime.

\section{ROTATION-BASED LIMITED FEEDBACK FRAMEWORKS}

We have argued the performance benefit of the random codebook-based differential feedback. However, it is impractical for a deployed system to employ a random codebook. In the following, we develop the systematic spherical cap codebook generation method introduced in Section II-D. In

\footnotetext{
${ }^{2}$ For matrices $\mathbf{A} \in \mathbb{C}^{n \times n}, \quad \mathbf{U} \in \mathbb{C}^{n \times k}, \quad \mathbf{C} \in \mathbb{C}^{k \times k}$, and $\quad \mathbf{V} \in \mathbb{C}^{k \times n}$ matrix inversion lemma states $(\mathbf{A}+\mathbf{U C V})^{-1}=\mathbf{A}^{-1}$. $\mathbf{A}^{-1} \mathbf{U}\left(\mathbf{C}^{-1}+\mathbf{V A}^{-1} \mathbf{U}\right)^{-1} \mathbf{V A}^{-1}$.
} 
Section IV-A, we first investigate a general rotation codebook $\mathcal{Q}$ design problem. In the absence of a straightforward design criterion for the rotation codebook $\mathcal{Q}$, we propose a capacity distortion minimizing rotation codebook design procedure for the independent block fading channel. Then, this general rotation codebook is extended to develop the differential feedback scheme in Section IV-B.

\section{A. Rotation Codebook Design}

In this subsection, consider a general capacity distortion minimizing rotation codebook design problem for the independent block fading channel. The optimal precoder $\overline{\mathbf{V}}_{m}$ is independent and isotropically distributed in $\mathcal{U}\left(M_{t}, M\right)$. This implies that without loss of generality, the optimal precoder $\overline{\mathbf{V}}_{m}$ can be modeled by $\overline{\mathbf{V}}_{m}=\boldsymbol{\Theta}_{m} \overline{\mathbf{V}}_{0}$ where $\boldsymbol{\Theta}_{m}$ is also isotropically distributed in $\mathcal{U}\left(M_{t}, M_{t}\right)$. For $m \geq 1$, then, the quantized precoder obeys the recursion $\mathbf{F}_{m}=\widehat{\widehat{\mathbf{\Theta}}}_{m} \overline{\mathbf{V}}_{0}$, where $\widehat{\boldsymbol{\Theta}}_{m}$ is chosen in $\mathcal{Q}=\left\{\boldsymbol{\Theta}_{i}\right\}_{i=1}^{2^{B}}$. To simplify the derivation, assume $\overline{\mathbf{V}}_{0}$ is known a priori to both the transmitter and receiver.

In the following, since we assume independent block-toblock fading in this subsection, the index $m$ is omitted. Setting $\mathbf{F}_{i}=\boldsymbol{\Theta}_{i} \overline{\mathbf{V}}_{0}$ and $\overline{\mathbf{V}}=\boldsymbol{\Theta} \overline{\mathbf{V}}_{0}$ and using (6) gives the distortion bound

$$
D(\mathcal{Q}) \leq \frac{\rho}{\ln (2)} E\left[\lambda_{1}^{2}\right] E\left[\min _{\boldsymbol{\Theta}_{i} \in \mathcal{Q}} d_{c}^{2}\left(\boldsymbol{\Theta} \overline{\mathbf{V}}_{0}, \boldsymbol{\Theta}_{i} \overline{\mathbf{V}}_{0}\right)\right] .
$$

To gain insight about how the rotation codebook is related to the throughput performance, we focus on the codebook dependent term $q \triangleq E\left[\min _{\boldsymbol{\Theta}_{i} \in \mathcal{Q}} d_{c}^{2}\left(\boldsymbol{\Theta} \overline{\mathbf{V}}_{0}, \boldsymbol{\Theta}_{i} \overline{\mathbf{V}}_{0}\right)\right]$ in (27), which can be rewritten and upper bounded by

$$
\begin{aligned}
q & =E\left[\min _{\boldsymbol{\Theta}_{i} \in \mathcal{Q}} \sum_{k=1}^{M}\left(1-\left\|\overline{\mathbf{V}}_{0}^{*} \boldsymbol{\Theta}^{*} \boldsymbol{\Theta}_{i} \mathbf{v}_{0, k}\right\|_{2}^{2}\right)\right] \\
& \leq E\left[\min _{\boldsymbol{\Theta}_{i} \in \mathcal{Q}} \sum_{k=1}^{M} 2\left(\left\|\overline{\mathbf{V}}_{0}^{*} \mathbf{v}_{0, k} e^{j \theta_{k}}\right\|_{2}-\left\|\overline{\mathbf{V}}_{0}^{*} \boldsymbol{\Theta}^{*} \boldsymbol{\Theta}_{i} \mathbf{v}_{0, k}\right\|_{2}\right)\right] \\
& \leq E\left[\min _{\boldsymbol{\Theta}_{i} \in \mathcal{Q}} \sum_{k=1}^{M} 2 \min _{\theta_{k}}\left\|\boldsymbol{\Theta} e^{j \theta_{k}}-\boldsymbol{\Theta}_{i}\right\|_{F}\right]
\end{aligned}
$$

where $\mathbf{v}_{0, k}$ denotes the $k$ th column of $\overline{\mathbf{V}}_{0}$. In the first bound, we use the fact $1-a^{2}=(1+a)(1-a)$ for $a=\left\|\overline{\mathbf{V}}_{0}^{*} \boldsymbol{\Theta}^{*} \boldsymbol{\Theta}_{i} \mathbf{v}_{0, k}\right\|_{2}$, apply a trivial bound $\left\|\overline{\mathbf{V}}_{0}^{*} \boldsymbol{\Theta}^{*} \boldsymbol{\Theta}_{i} \mathbf{v}_{0, k}\right\|_{2} \leq 1$ to the $(1+a)$ term, and use the fact that $\left\|\overline{\mathbf{V}}_{0}^{*} \mathbf{v}_{0, k} e^{j \theta_{k}}\right\|_{2}=1$ for the $(1-a)$ term where the $e^{j \theta_{k}}$ is used to minimize the distortion. By optimizing over $\theta_{k}$, (28) yields

$$
q \leq 2 M \sqrt{2 M_{t}} E\left[\min _{\Theta_{i} \in \mathcal{Q}} \sqrt{1-\frac{1}{M_{t}}\left|\operatorname{tr}\left(\boldsymbol{\Theta}^{*} \boldsymbol{\Theta}_{i}\right)\right|}\right] .
$$

Fully motivated by (29), we define a distance between two unitary matrices $\boldsymbol{\Theta} \in \mathcal{U}\left(M_{t}, M_{t}\right)$ and $\boldsymbol{\Theta}_{i} \in \mathcal{U}\left(M_{t}, M_{t}\right)$ as

$$
d\left(\boldsymbol{\Theta}_{i}, \boldsymbol{\Theta}_{j}\right)=\sqrt{1-\frac{1}{M_{t}}\left|\operatorname{tr}\left(\boldsymbol{\Theta}_{i}^{*} \boldsymbol{\Theta}_{j}\right)\right|} .
$$

Before proceeding, we must show that (30) is a valid metric.

Theorem 1: The function $d\left(\boldsymbol{\Theta}_{i}, \boldsymbol{\Theta}_{j}\right)=\sqrt{1-\frac{1}{M_{t}}\left|\operatorname{tr}\left(\boldsymbol{\Theta}_{i}^{*} \boldsymbol{\Theta}_{j}\right)\right|}$ is a metric in $\mathcal{U}\left(M_{t}, M_{t}\right)$.

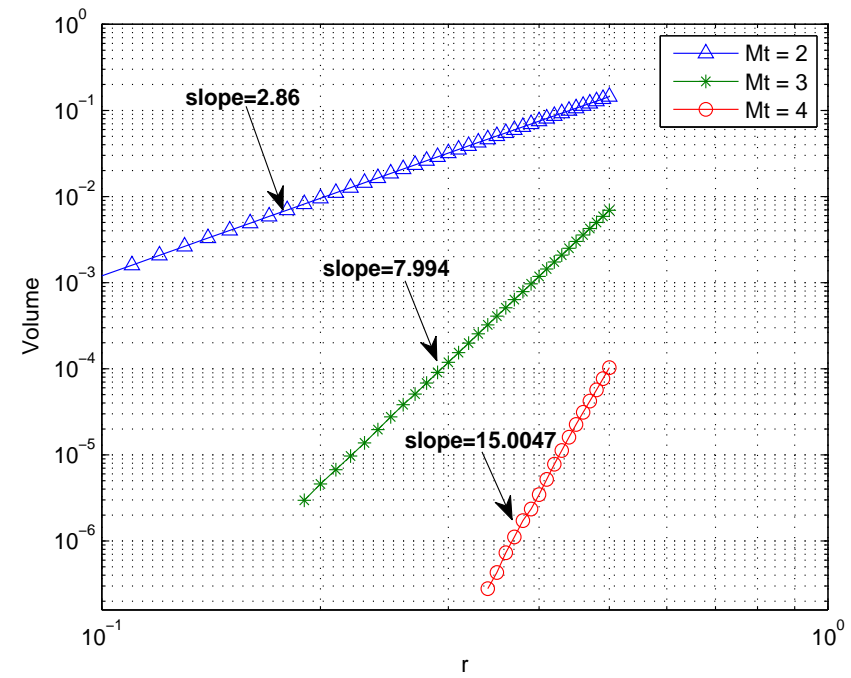

Fig. 2. Volume estimation for the rotation codebook space, $M_{t}=2,3,4$.

Proof: See Appendix VII-C.

In what follows, the rotation codebook space is characterized, which enables us to take the density of the codebook $\mathcal{Q}$ into account when solving the capacity distortion minimization problem.

1) Rotation Codebook Space: The bound (29) implies that the capacity distortion is related to the minimum distance. Define a single dimensional rotation matrix as $\mathbf{C}_{\theta}=e^{j \theta} \mathbf{I}_{M_{t}}$. In $D(\mathcal{Q})$, right (or left) multiplying $\mathbf{C}_{\theta}$ to $\boldsymbol{\Theta}_{i}$ does not change the distortion and thereby does not alter the bound in (29). For example, this means that if $\Theta_{1}$ is the codeword that maximizes the mutual information, then modifying $\boldsymbol{\Theta}_{1} \mathbf{C}_{\theta}$ gives the same mutual information. Therefore, the transmission is $\mathbf{C}_{\theta}$ rotationally invariant. Define the set $\mathcal{R}\left(M_{t}, M_{t}\right)=\left\{\mathbf{C}_{\theta}\right.$ : $0 \leq \theta<2 \pi\}$. Since $\mathcal{R}\left(M_{t}, M_{t}\right)$ is a subgroup of the unitary group $\mathcal{U}\left(M_{t}, M_{t}\right)$, by the equivalence relation $\Theta \sim \mathbf{\Theta C}_{\theta}$, the rotation codebook space (which is a quotient space) is represented by $\mathcal{Z}\left(M_{t}, M_{t}\right)=\mathcal{U}\left(M_{t}, M_{t}\right) / \mathcal{R}\left(M_{t}, M_{t}\right)$. It has been shown that this kind of quotient space is a Riemannian manifold [40]. Thus, the distance $d\left(\boldsymbol{\Theta}_{i}, \boldsymbol{\Theta}_{j}\right)$ in (30) is defined in the Riemannian manifold $\mathcal{Z}\left(M_{t}, M_{t}\right)$. Since $\operatorname{dim}\left(\mathcal{R}\left(M_{t}, \mathrm{M}\right)\right)=1$ where $\operatorname{dim}(\mathcal{A})$ extracts the dimension of the space $\mathcal{A}$, by the dimension theorem of a quotient space [41], $\operatorname{dim}\left(\mathcal{Z}\left(M_{t}, M_{t}\right)\right)=M_{t}^{2}-1$. This analysis allows us to measure a volume of a metric ball in $\mathcal{Z}\left(M_{t}, M_{t}\right)$.

An open ball of radius $r$ centered at $\boldsymbol{\Theta}_{i}$ is defined by $\mathcal{B}_{\boldsymbol{\Theta}_{i}}=$ $\left\{\boldsymbol{\Theta} \in \mathcal{Z}\left(M_{t}, M_{t}\right): d\left(\boldsymbol{\Theta}, \boldsymbol{\Theta}_{i}\right) \leq r\right\}$. For large $M_{t}$, it has been shown in [40], [42] that

$$
\operatorname{vol}\left(\mathcal{B}_{\Theta}(r)\right) \approx C_{M_{t}} r^{M_{t}^{2}-1},
$$

where $C_{M_{t}}$ is a constant only depending on $M_{t}$. To ensure (31), Fig. 2 shows the volume estimation result of (31) in $\mathcal{Z}\left(M_{t}, M_{t}\right)$, which is measured by (30). To show the slope, we take the logarithm of the volume and radius. As can be seen from Fig. 2, the slope $M_{t}^{2}-1$ is well estimated by (31). Now, we use the formula in (31) to obtain the rotation codebook design criterion. 
2) Rotation codebook design criterion: The minimum distance between two codewords in $\mathcal{Q}$ is defined by $\delta(\mathcal{Q})=\min _{1 \leq l<k \leq K} d\left(\boldsymbol{\Theta}_{l}, \boldsymbol{\Theta}_{k}\right)$. Note that if $r \leq \frac{\delta(\mathcal{Q})}{2}, \mathcal{B}_{\boldsymbol{\Theta}_{l}}(r) \cap$ $\mathcal{B}_{\Theta_{k}}(r)=\phi$ for $k \neq l$. Then, a density of $\mathcal{Q}$ characterized by $\delta(\mathcal{Q})$ is given

$$
\boldsymbol{\Delta}(\mathcal{Q})=\frac{\operatorname{vol}\left(\bigcup_{i=1}^{K} \mathcal{B}_{\boldsymbol{\Theta}_{i}}(\delta(\mathcal{Q}) / 2)\right)}{\operatorname{vol}\left(\mathcal{Z}\left(M_{t}, M_{t}\right)\right)}=\frac{K \cdot \operatorname{vol}\left(\mathcal{B}_{\boldsymbol{\Theta}_{1}}(\delta(\mathcal{Q}) / 2)\right)}{\operatorname{vol}\left(\mathcal{B}_{\boldsymbol{\Theta}}(1)\right)} .
$$

Using (31), $\Delta(\mathcal{Q})$ can be approximated by

$$
\boldsymbol{\Delta}(\mathcal{Q}) \approx K\left(\frac{\delta(\mathcal{Q})}{2}\right)^{M_{t}^{2}-1} .
$$

Now, using the approach in [43], we can relate the average distortion incurred by the rotation codebook $\mathcal{Q}$ to the codebook density $\boldsymbol{\Delta}(\mathcal{Q})$ as

$$
\begin{aligned}
E\left[\min _{\boldsymbol{\Theta}_{i} \in \mathcal{Q}} d\left(\boldsymbol{\Theta}, \boldsymbol{\Theta}_{i}\right)\right] \leq & \operatorname{Pr}\left(\min _{\boldsymbol{\Theta}_{i} \in \mathcal{Q}} d\left(\boldsymbol{\Theta}, \boldsymbol{\Theta}_{i}\right) \leq \frac{\delta(\mathcal{Q})}{2}\right) \frac{\delta(\mathcal{Q})}{2} \\
& +\operatorname{Pr}\left(\min _{\boldsymbol{\Theta}_{i} \in \mathcal{Q}} d\left(\boldsymbol{\Theta}, \boldsymbol{\Theta}_{i}\right)>\frac{\delta(\mathcal{Q})}{2}\right) \\
\approx & {\left[1+K\left(\frac{\delta(\mathcal{Q})}{2}\right)^{M_{t}^{2}-1}\left(\frac{\delta(\mathcal{Q})}{2}-1\right)\right] . }
\end{aligned}
$$

Consequently, it can be readily shown that minimizing (32) is equivalent to maximizing $\delta(\mathcal{Q})$. The rotation codebook design criterion follows:

$$
\mathcal{Q}=\underset{\widetilde{\mathcal{Q}}}{\operatorname{argmax}} \delta(\widetilde{\mathcal{Q}}) .
$$

\section{B. Extension to Rotation-Based Differential Feedback}

After designing $\mathcal{Q}$, our goal is to develop a systematic spherical cap codebook adaptation strategy. As aforementioned in Section I, our approach for obtaining the adaptive spherical cap codebook is based on perturbing $\mathbf{F}_{m-1}$ in Euclidean space (using $\mathcal{Q}$ ) and projecting the perturbed matrices onto $\mathcal{U}\left(M_{t}, M\right)$.

In our approach, perturbation around $\mathbf{F}_{m-1}$ is generated using $\boldsymbol{\Theta}_{i} \in \mathcal{Q}$ designed by (33). To ease the codebook generation, given $r_{m}^{2}$ in (17), we define the normalized squared spherical cap radius as

$$
\bar{r}_{m}^{2}=r_{m}^{2} / \min \left\{M, M_{t}-M\right\}
$$

where $0 \leq \bar{r}_{m}^{2} \leq 1$. Note that $\mathcal{Q}$ does not depend on the number of transmit streams $M$. A single rotation codebook $\mathcal{Q}$ can be used for any transmission rank $\left(1 \leq M \leq M_{t}-\right.$ 1 ). Depending on the method of generating the perturbations, we consider two possible spherical cap codebook adaptation strategies.

1) Method 1: Perturbation in Euclidean Space $\mathbb{C}^{M_{t} \times M}$ : Given the previous precoder $\mathbf{F}_{m-1}, \mathbf{F}_{m-1}$ is perturbed according to

$$
\mathbf{\Psi}_{\bar{r}_{m}, i}=w_{m} \mathbf{F}_{m-1}+\bar{r}_{m} \boldsymbol{\Theta}_{i} \mathbf{F}_{m-1}
$$

where $\bar{r}_{m}$ is the normalized spherical cap radius in (34), $\boldsymbol{\Theta}_{i} \in \mathcal{Q}$ is a rotation codeword, and $w_{m}\left(0 \leq w_{m} \leq 1\right)$ is a free parameter for adaptation. The rotation matrix $\boldsymbol{\Theta}_{i}$ determines the direction of the perturbation added to $w_{m} \mathbf{F}_{m-1}$ and $\bar{r}_{m}$ defines its amount. Iterating (35) from $i=1$ to $2^{B}$, a set of perturbations $\left\{\boldsymbol{\Psi}_{\bar{r}_{m}, i}\right\}_{i=1}^{2^{B}}$ is generated. Note that $\boldsymbol{\Psi}_{\bar{r}_{m}, i} \in \mathbb{C}^{M_{t}, M}$. The adaptation of $\left\{\boldsymbol{\Psi}_{\bar{r}_{m}, i}\right\}_{i=1}^{2^{B}}$ to the precoding codebook $\mathcal{F}_{m}=\left\{\mathbf{F}_{m, i}\right\}_{i=1}^{2^{B}}$ is done by projecting $\boldsymbol{\Psi}_{\bar{r}_{m}, i}$ onto $\mathcal{U}\left(M_{t}, M\right)$. Denote the orthonormally projected matrix as $\operatorname{proj}\left(\boldsymbol{\Psi}_{\bar{r}_{m}, i}\right)$ and

$$
\mathbf{F}_{m, i}=\operatorname{proj}\left(\mathbf{\Psi}_{\bar{r}_{m}, i}\right) .
$$

Either Procrustes orthonormalization [44] or Gram-Schmidt column orthonormalization [44] can be used as the projection function. If we denote the compact SVD of $\boldsymbol{\Psi}_{\bar{r}_{m}, i}$ as $\boldsymbol{\Phi}_{i} \mathbf{D}_{i} \boldsymbol{\Pi}_{i}^{*}$, where $\boldsymbol{\Phi}_{i} \in \mathcal{U}\left(M_{t}, M\right), \boldsymbol{\Pi}_{i} \in \mathcal{U}(M, M)$, and $\mathbf{D}_{i} \in \mathbb{C}^{M \times M}$ is the singular value matrix of $\boldsymbol{\Psi}_{\bar{r}_{m}, i}$, the solution to the Procrustes problem is given by $\mathbf{F}_{m, i}=\boldsymbol{\Phi}_{i} \boldsymbol{\Pi}_{i}^{*}$ [44] and Gram-Schmidt column orthonormalization returns $\mathbf{F}_{m, i}=\boldsymbol{\Phi}_{i}$. Note that both projection methods ultimately give the same performance because $\boldsymbol{\Phi}_{i} \boldsymbol{\Pi}_{i}^{*}$ and $\boldsymbol{\Phi}$ are in the equivalent relation in Grassmannian manifold (i.e., $\boldsymbol{\Phi}_{i} \boldsymbol{\Pi}_{i}^{*} \sim \boldsymbol{\Phi}_{i}$ ). For simplicity, we just use Gram-Schmidt column orthonormalization.

Note that the precoding codebook $\mathcal{F}_{m}$ acquired by solving (35) and (36) does not guarantee that $\mathcal{F}_{m} \subset \mathcal{S}_{\mathbf{F}_{m-1}}\left(r_{m}\right)$. We need to design $w_{m}$ so that $\mathcal{F}_{m} \subset \mathcal{S}_{\mathbf{F}_{m-1}}\left(r_{m}\right)$.

Theorem 2: The adaptive codebook $\mathcal{F}_{m}$ obtained by (35) and (36) resides in $\mathcal{S}_{\mathbf{F}_{m-1}}\left(r_{m}\right)$ if $w_{m}=\sqrt{1-\bar{r}_{m}^{2}}$.

Proof: See Appendix VII-D.

Thus, the function $\vartheta$ in (9) is explicitly described by $\mathbf{F}_{m, i}=\operatorname{proj}\left(\sqrt{1-\bar{r}_{m}} \mathbf{F}_{m-1}+\bar{r}_{m} \boldsymbol{\Theta}_{i} \mathbf{F}_{m-1}\right)$.

2) Method 2: Perturbation in Euclidean Space $\mathbb{C}^{M_{t} \times M_{t}}$ : In Method 1 , the codebook adaptation must be done at runtime because procedures in (35) and (36) require a priori $\mathbf{F}_{m-1}$. It is practically advantageous to design the codebook offline as a function of the channel statistics. For this objective, we propose an adaptive rotation codebook design scheme independent of $\mathbf{F}_{m-1}$.

The perturbation $\boldsymbol{\Psi}_{\bar{r}_{m}, i}$ in (35) is obtained by transforming $\mathbf{F}_{m-1}$ via the matrix

$$
\mathbf{R}_{\bar{r}_{m}, i}=\sqrt{1-\bar{r}_{m}^{2}} \mathbf{I}_{M_{t}}+\bar{r}_{m} \boldsymbol{\Theta}_{i},
$$

i.e., $\boldsymbol{\Psi}_{\bar{r}_{m}, i}=\mathbf{R}_{\bar{r}_{m}, i} \mathbf{F}_{m-1}$. Note that $\mathbf{R}_{\bar{r}_{m}, i} \in \mathbb{C}^{M_{t} \times M_{t}}$. Since $\mathbf{R}_{\bar{r}_{m}, i}$ is a linear combination of the unitary matrices $\mathbf{I}_{M_{t}}$ and $\boldsymbol{\Theta}_{i}$, the subspace of $\mathbf{R}_{\bar{r}_{m}, i}$ lies within the subspace spanned by $\mathbf{I}_{M_{t}}$ and $\boldsymbol{\Theta}_{i}$. Thus, orthonormally projecting $\mathbf{R}_{\bar{r}_{m}, i}$ back to $\mathcal{U}\left(M_{t}, M_{t}\right)$ according to

$$
\boldsymbol{\Theta}_{m, i}=\operatorname{proj}\left(\mathbf{R}_{\bar{r}_{m}, i}\right)
$$

produces a rotation codeword that lies within the subspace spanned by $\mathbf{I}_{M_{t}}$ and $\Theta_{i}$. In this way, the adaptive rotation codebook $\mathcal{Q}_{m}=\left\{\boldsymbol{\Theta}_{m, i}\right\}_{i=1}^{2^{B}}$ is generated by projecting the perturbation $\mathbf{R}_{\bar{r}_{m}, i}$ for $i=1, \ldots, 2^{B}$.

Given $\mathbf{F}_{m-1}$, the $i$ th precoding codeword at the $m$ th channel instance is represented by $\mathbf{F}_{m, i}=\boldsymbol{\Theta}_{m, i} \mathbf{F}_{m-1}$. The best precoder $\mathbf{F}_{m}$ is given by finding $\boldsymbol{\Theta}_{m}=\operatorname{argmax} I\left(\boldsymbol{\Theta}_{m, i} \mathbf{F}_{m-1}\right)$ $\boldsymbol{\Theta}_{m, i} \in \mathcal{Q}_{m}$

and setting $\mathbf{F}_{m}=\boldsymbol{\Theta}_{m} \mathbf{F}_{m-1}$. As before, either Proscrustes orthonormalization or Gram-Schmidt orthonormalization can be employed for the projection. We assume Gram-Schmidt 


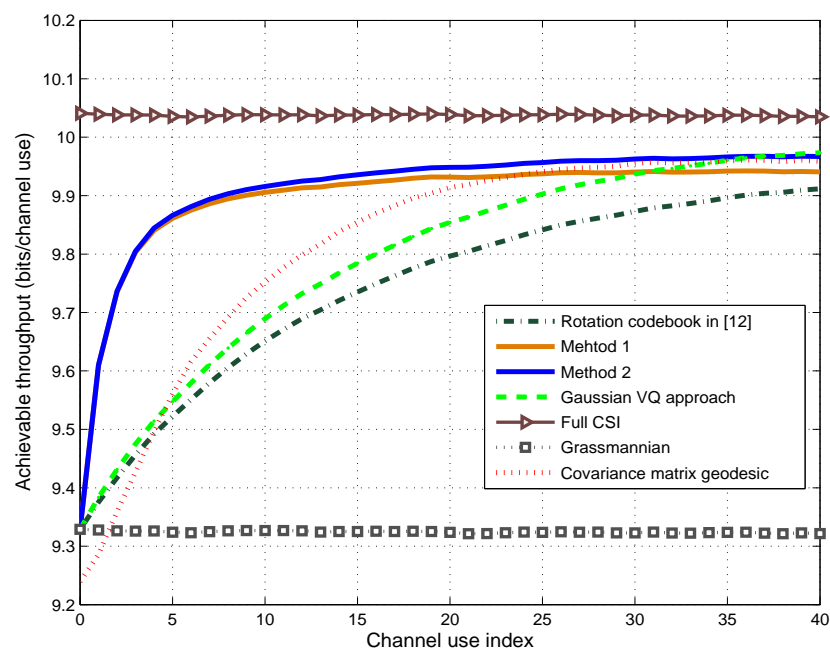

Fig. 3. Achievable throughput vs. channel use index for $M_{t}=M_{r}=4$, $M=2, B=4$ bits, $S N R=10 d B$, and $v=1 \mathrm{~km} / \mathrm{h}(\epsilon=0.999)$.

column orthonormalization because it is efficiently obtained by a Gram-Schmidt QR decomposition (or other fast QR algorithms) and it shows robustness in tracking capability [7], [30].

The function $\vartheta$ in (9) is now explicitly depicted by $\mathbf{F}_{m, i}=\operatorname{proj}\left(\sqrt{1-\bar{r}_{m}} \mathbf{I}_{M_{t}}+\bar{r}_{m} \boldsymbol{\Theta}_{i}\right) \mathbf{F}_{m-1}$. In Method 2, the evolution of $\mathcal{Q}_{m}$ solely depends on the evolution of $\bar{r}_{m}$. From (17), as $m$ tends infinity, $\bar{r}_{m}^{2}$ converges to

$$
\bar{r}_{m}^{2} \stackrel{m \rightarrow \infty}{=}(1-\epsilon)^{2}\left(\frac{M\left(M_{t}-M\right) / M_{t}}{\min \left(M, M_{t}-M\right)}\right)\left(\frac{1}{1-\epsilon^{2} 2^{-\frac{B}{M\left(M_{t}-M\right)}}}\right)
$$

indicating for every $\delta>0$ there always exists an integer $N$ such that $m \geq N$ implies $\left|\bar{r}_{m+1}-\bar{r}_{m}\right| \leq \delta$. This observation suggests that in a practical system given a threshold $\delta>0$, a finite set of adaptive rotation codebooks $\mathcal{Q}_{1}, \ldots, \mathcal{Q}_{N}$ is employed for the first $N$ channel instances and for $m>N, \mathcal{Q}_{N}$ is constantly used. This indicates that system can efficiently use a predefined codebook set $\left\{\mathcal{Q}_{l}\right\}_{l=0}^{N}$ and avoid run-time computation for the rotation codebook evolution (details are discussed in the next section).

\section{Simulations and Discussions}

In this section, we perform Monte Carlo simulations to investigate the achievable throughput performance of the proposed schemes in slowly varying MIMO channels. First, to ensure the operation of the proposed scheme, we discuss the achievable throughput with the first-order Gauss-Markov channel model in (2). Second, to evaluate channel model mismatch and to provide a practical intuition about the performance, we employ the spatial channel model (SCM) [45] which is officially used to evaluate the throughput performance of standards such as IEEE 802.16m [23] and 3GPP LTE [24].

Throughout the simulation study, we assume $M_{t}=M_{r}=4$ and $M=2$ MIMO spatial multiplexing system and fix $B=4$ bits which is justified and motivated by practical standards [23], [24]. To quantize $\mathbf{F}_{0}$ in the proposed differential feedback

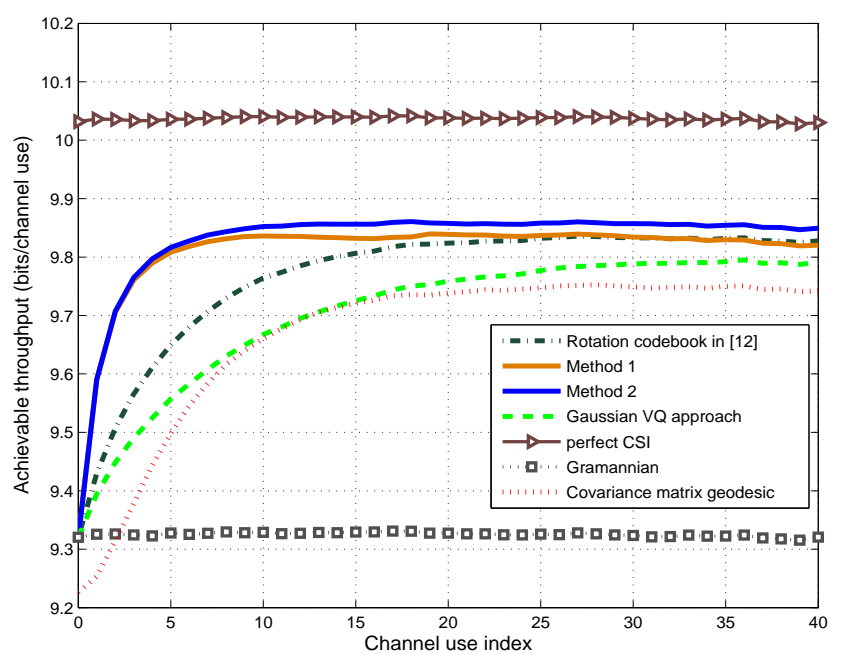

Fig. 4. Achievable throughput vs. channel use index for $M_{t}=M_{r}=4$, $M=2, B=4$ bits, $S N R=10 \mathrm{~dB}$, and $v=3 \mathrm{~km} / \mathrm{h}(\epsilon=0.988)$.

scheme we use a Grassmannian subspace packing (GSP) codebook [2]. The throughput performance of the Gaussian VQ approach in [8] is also simulated. Note that in [8], the initial precoder is set to $\mathbf{F}_{0}=\mathbf{I}_{1: M}$ where $\mathbf{I}_{1: M}$ denotes the matrix formed by taking the first $M$ columns of identity matrix $\mathbf{I}_{M_{t}}$. To provide a fair comparison, we modify [8] to also use the GSP codebook to quantize the initial precoder and apply the capacity selection criterion in (4). The eighthorder polynomial in [8] is employed to optimize $a$ in (7). We also simulate the covariance matrix geodesic approach in [25]. Note that the technique in [25] is different than both our approach and the Gaussian VQ approach in [8] because it allows a waterfilling-based transmit covariance design. In [25], the initial covariance matrix is set to $\mathbf{I}_{M_{t}}$. We also modify [25] to use a $B=4$ bit covariance codebook at the initial state so that the initial quantized covariance matrix is selected to maximize the mutual information evaluated by designing waterfilling percoder for each covariance codeword. In [25], assuming the first-order Gauss-Markov channel model, adaptation to the channel correlation is done by adjusting step size $\Delta$ parameter which must be found using Monte-Carlo simulation. Given the $\epsilon$ value in (2), a blind search is applied to optimize $\Delta$.

As aforementioned in Section II-A, $\epsilon$ follows Jake's model [34] (i.e., $\epsilon=J_{0}\left(2 \pi f_{D} T\right)$ ). When we generate $\epsilon$, system parameters employed in IEEE $802.16 \mathrm{~m}$ standard [23] are used. In IEEE $802.16 \mathrm{~m}$, closed-loop operation assumes $3 \mathrm{~km} / \mathrm{h}$ velocity, feedback interval of $5 \mathrm{~ms}$, and $f_{c}=2.5 \mathrm{GHz}$, where the typical time correlation coefficient is $\epsilon=0.988$. The $\epsilon$ varies from 0.999 to 0.872 as the terminal speed varies from 1 $\mathrm{km} / \mathrm{h}$ to $10 \mathrm{~km} / \mathrm{h}$. Fig. 3, 4, 5, and 6 display the achievable throughput with respect to the channel use index for $v=1$ $\mathrm{km} / \mathrm{h}(\epsilon=0.999), v=3 \mathrm{~km} / \mathrm{h}(\epsilon=0.988), v=7 \mathrm{~km} / \mathrm{h}$ $(\epsilon=0.936)$, and $v=10 \mathrm{~km} / \mathrm{h}(\epsilon=0.872)$ in the firstorder Gauss-Markov channel. The SNR is fixed at $10 \mathrm{~dB}$. In the figures, 'Method 1' and 'Method 2' denote the proposed 


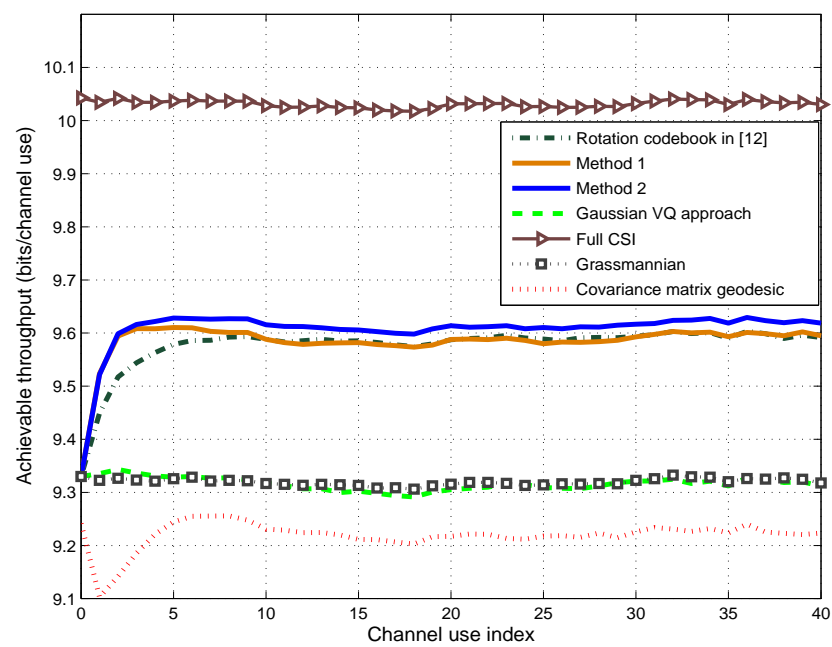

Fig. 5. Achievable throughput vs. channel use index for $M_{t}=M_{r}=4$, $M=2, B=4$ bits, $S N R=10 d B$, and $v=7 \mathrm{~km} / \mathrm{h}(\epsilon=0.936)$.

schemes in Section IV-B1 and Section IV-B2, respectively. The 'Gaussian VQ approach' and 'Covariance matrix geodesic' indicate the differential feedback scheme in [8] and [25], respectively. The performance of the rotation codebook in [14] is also evaluated. The differential schemes in [8] and [25] fail to track the channel variation when $v \geq 7 \mathrm{~km} / \mathrm{h}$. The tracking performance of the proposed schemes outperforms the other schemes and shows fast convergence to the steadystate. Note that the gain of our schemes mainly comes from the refinement of the spherical cap radius $r_{m}$. Compared to [14], accounting the channel directional variation and quantization error propagation into $r_{m}$ design gives sufficient improvement on the tracking performance at the initial stages. The initial improvement of the tracking performance is the crucial factor that seems to determine the robustness of the differential feedback schemes because in practical systems a differential feedback with periodic reset is used. For instance, in IEEE $802.16 \mathrm{~m}$ and LTE-Advanced, the differential feedback is reinitiated every $15 \mathrm{~ms}$ to $30 \mathrm{~ms}$ period (i.e., every $m=3$ to $m=6$ ). Reset is done by using non-differential feedback (e.g., using a GSP codebook). This refreshment is important because the channel correlation statistic often changes within a refreshment period and feedback delay can also contaminate the differential performance.

The drawbacks of [25] are that the codebook size is always constrained such that $2^{B} \leq M_{t}^{2}$ and the geodesic model on the positive definite covariance matrix space is valid when $M_{r} \geq M_{t}$. Also, whenever the system changes its configurations (e.g., $M_{t}, M_{r}, M$, and $B$ ), $\Delta$ must be redesigned using Monte-Carlo simulation, while in our scheme $r_{m}$ is conveniently modified according to (17). Compared to [8] and our scheme, the approach in [25] requires huge run-time complexity. For example, in order to construct the quantized waterfilling precoder at each channel instance, it requires $2^{B}$ Gram-Schmidt orthonormalizations in $M_{t}^{2}$ dimensional space, $2^{B-1}$ SVDs to derive geodesic curves, $2^{B}$ matrix inversions to

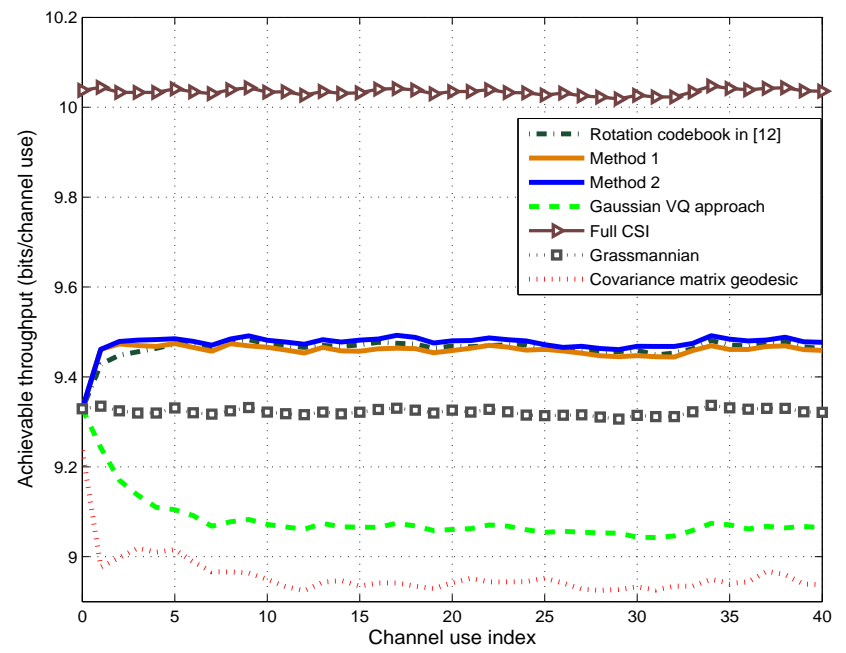

Fig. 6. Achievable throughput vs. channel use index for $M_{t}=M_{r}=4$, $M=2, B=4$ bits, $S N R=10 d B$, and $v=10 \mathrm{~km} / \mathrm{h}(\epsilon=0.872)$.

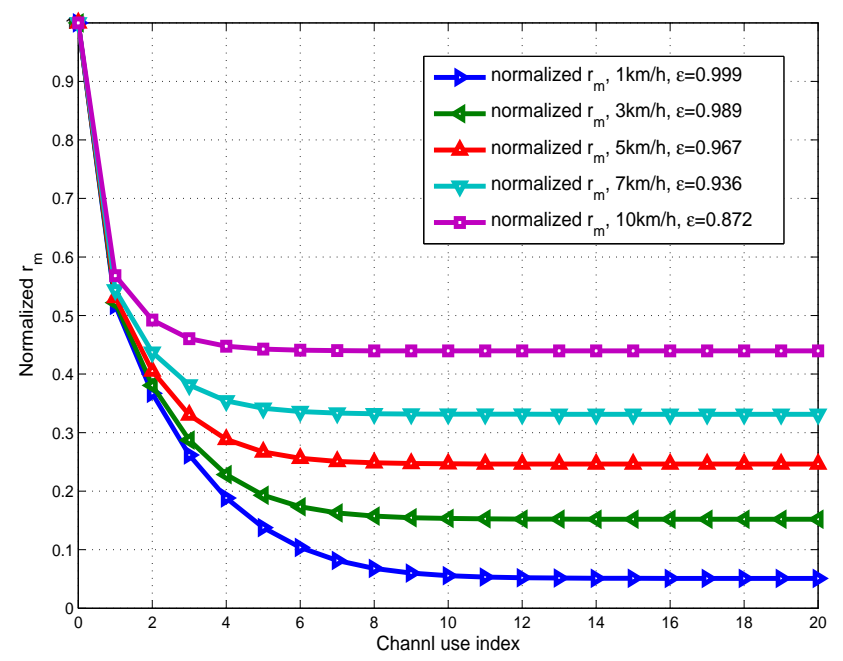

Fig. 7. Normalized spherical cap radius $\bar{r}_{m}$ with $M_{t}=M_{r}=4, M=2$, and $B=4$ bits.

select the best quantized covariance matrix, and one execution of waterfilling optimization. On the other hand, the proposed scheme requires $2^{B}$ Gram-Schmidt orthonormalizations in $M_{t}$ dimensional space and $2^{B}$ matrix determinants. In terms of required complexity, [8] shows the lowest computational overhead only requiring $2^{B}$ matrix determinants to search for the precoder.

Next, we plot the evolution of the normalized spherical cap radius $\bar{r}_{m}$ in (17) and show that the complexity of Method 2 can be efficiently decreased by excluding the run-time GramSchmidt orthonormalization step. Fig. 7 shows $\bar{r}_{m}$ for different values of $\epsilon$. As it can be seen from the Fig. 7, $\bar{r}_{m}$ tends to be small as the amount of correlation increases. Also, Fig. 7 reveals the convergence (39). The value of $\bar{r}_{m}$ converges to its steady state value $\bar{r}_{\infty}$ rapidly. For example, given the threshold $\delta=10^{-3}$, the minimum integer $N$ ensuring that 


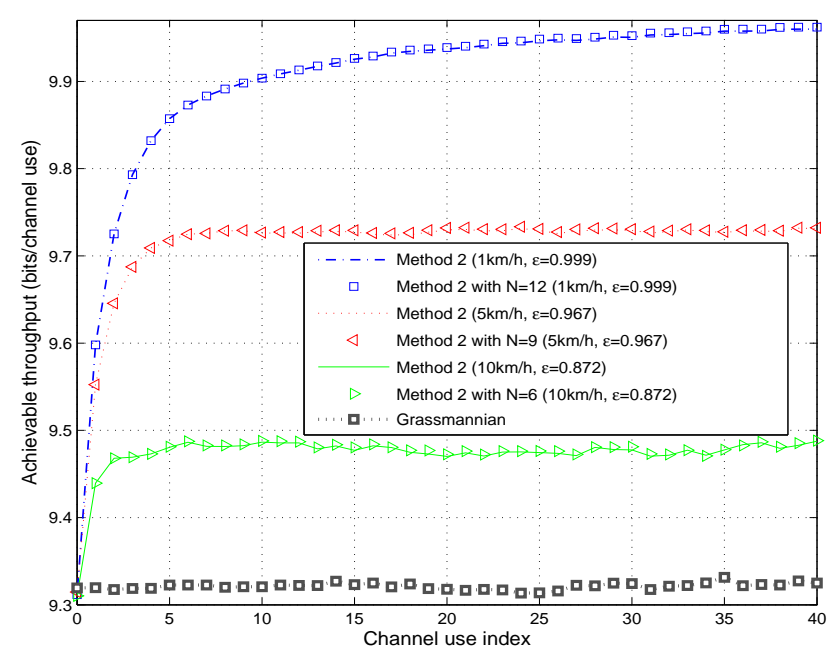

Fig. 8. Achievable throughput of Method 2 with finite rotation codebook evolution and infinite rotation codebook evolution for $M_{t}=M_{r}=4, M=$ $2, B=4$ bits, and $S N R=10 d B$.

$m \geq N$ implies $\left|\bar{r}_{m+1}-\bar{r}_{m}\right| \leq \delta$ is given by $N=12$ for $v=1$ $\mathrm{km} / \mathrm{h}, N=10$ for $v=3 \mathrm{~km} / \mathrm{h}, N=8$ for $v=7 \mathrm{~km} / \mathrm{h}$, and $N=6$ for $v=10 \mathrm{~km} / \mathrm{h}$. This demonstrates that when Method 2 in Section IV-B2 is used, the system only needs a finite sequence of rotation codebooks $\mathcal{Q}_{1}, \ldots, \mathcal{Q}_{N}$ designed offline. Fig. 8 displays the throughput performances of Method 2 obtained by the finite rotation codebook evolution (with $\delta=$ $10^{-3}$ ) and infinite rotation codebook evolution. For example, when $v=5 \mathrm{~km} / \mathrm{h}$, Method 2 with finite rotation codebook evolution performs differential feedback for the first $N=9$ times of channel uses and for $m>9$, the rotation codebook is set constantly to $\mathcal{Q}_{m}=\mathcal{Q}_{9}$. As can be seen from Fig. 8, the throughput difference between the finite and infinite codebook evolutions is negligible. One of the benefits of the rotation approach is that the codebook is designed independently of the rank. Hence a single finite sequence rotation codebook can be used regardless of the rank. The algorithm in [8] stores SVD results for all codewords to avoid computing $2^{B}$ different SVDs when deriving the geodesic curves. For one codeword, it stores 3 different matrices. Since there are $M_{t}-1$ codebooks for supporting $M_{t}-1$ different ranks, [8] requires storage of a total of $3\left(M_{t}-1\right) 2^{B}$ matrices. When $M_{t}=4$ and $v=5$ $\mathrm{km} / \mathrm{h}$, Method 2 with $\delta=10^{-3}$ requires storage of $10 \cdot 2^{B}$ matrices and the Gaussian VQ approach requires storage of $9 \cdot 2^{B}$ matrices. Though Method 2 still needs larger storage than that of the Gaussian VQ approach, the benefit comes from the drastic throughput improvement.

Finally, we examine the throughput performance of the proposed differential feedback frameworks with channel model mismatch. To examine this, SCM [45] is employed. Since the closed-loop MIMO operation assumes $v=3 \mathrm{~km} / \mathrm{h}$ mobility in IEEE $802.16 \mathrm{~m}$ [23], the evaluation is performed with $v=3$ $\mathrm{km} / \mathrm{h}$. The antenna spacing at the transmitter and receiver is set to four wavelengths separation with an angle spread of 15 degrees. We assume an Urban Macro scenario. Fig.

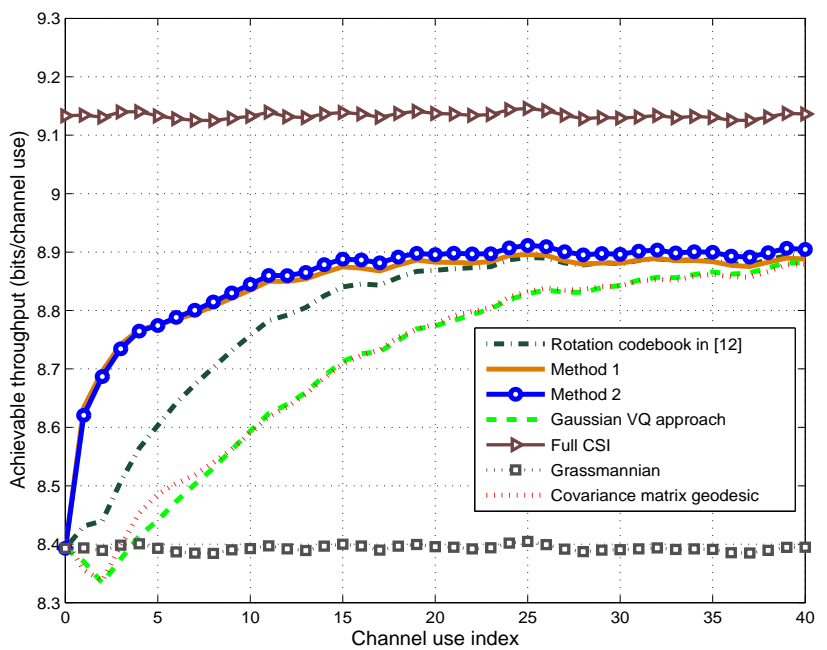

Fig. 9. Achievable throughput vs. channel use index for SCM with $M_{t}=$ $M_{r}=4, M=2, B=4$ bits, $S N R=10 \mathrm{~dB}$, and $v=3 \mathrm{~km} / \mathrm{h}(\epsilon=$ $0.988)$.

9 compares the proposed differential feedback with other techniques in [8] and [25]. In addition, we also plot the finite codebook evolution with $N=10$. As can be seen from Fig. 9, the throughput difference between Method 2 and Method 2 with $N=10$ is negligible. Fig. 9 and Fig. 4 both show the same performance trends for nearly all algorithms. The Gaussian VQ approach and covariance matrix geodesic suffer from performance degradations at the beginning stages of differential operations.

\section{CONCLUSIONS}

We proposed frameworks for performing limited feedback spatial multiplexing over a temporally correlated MIMO channel using a rotation-based differential codebook adaptations. We quantified the average directional variation and developed a recursive formula for the spherical cap radius using the random matrix quantization arguments. Based on these statistics, the throughput performance was analyzed. To develop the systematic differential feedback adaptation schemes, a general rotation codebook design problem was investigated, and we extended this rotation codebook to construct a spherical cap codebook where the cap radius is controlled according to the statistic of the directional variation. Two spherical cap codebook-based differential feedback schemes were proposed depending on the method of generating the perturbation set. From the simulation study, the proposed framework showed significant throughput gain in slow fading channel environment.

\section{APPENDIX}

\section{A. Proof of (11)}

The first term $E\left[\left\|\mathbf{H}_{m} \overline{\mathbf{V}}_{m}\right\|_{F}^{2}\right]=E\left[\operatorname{tr}\left(\overline{\boldsymbol{\Sigma}}_{m}^{2}\right)\right]$ on the left hand side (1.h.s.) of (11) can be equivalently rewritten

$$
E\left[\operatorname{tr}\left(\overline{\boldsymbol{\Sigma}}_{m}^{2}\right)\right]=\epsilon^{2} E\left[\operatorname{tr}\left(\overline{\boldsymbol{\Sigma}}_{m-1}^{2}\right)\right]+\left(1-\epsilon^{2}\right) E\left[\operatorname{tr}\left(\overline{\boldsymbol{\Lambda}}_{m}^{2}\right)\right]
$$




$$
\begin{aligned}
E\left[\left\|\mathbf{H}_{m} \mathbf{V}_{m}\right\|_{F}^{2}-\left\|\mathbf{H}_{m} \mathbf{F}_{m-1}\right\|_{F}^{2}\right] & \leq E\left[\operatorname{tr}\left(\epsilon^{2} \overline{\mathbf{\Sigma}}_{m-1}^{2}\left(\mathbf{I}_{M}-\overline{\mathbf{V}}_{m-1}^{*} \mathbf{F}_{m-1} \mathbf{F}_{m-1}^{*} \overline{\mathbf{V}}_{m-1}\right)+\left(1-\epsilon^{2}\right) \overline{\mathbf{\Lambda}}_{m}^{2}\left(\mathbf{I}_{M}-\overline{\mathbf{P}}_{m}^{*} \mathbf{F}_{m-1} \mathbf{F}_{m-1}^{*} \overline{\mathbf{P}}_{m}\right)\right)\right] \\
& \leq E\left[\operatorname{tr}\left(\overline{\mathbf{\Sigma}}_{m}^{2}\right)\right] E\left[\epsilon^{2}\left(M-\left\|\overline{\mathbf{V}}_{m-1}^{*} \mathbf{F}_{m-1}\right\|_{F}^{2}\right)+\left(1-\epsilon^{2}\right)\left(M-\left\|\overline{\mathbf{P}}_{m}^{*} \mathbf{F}_{m-1}\right\|_{F}^{2}\right)\right]
\end{aligned}
$$

where $\overline{\boldsymbol{\Lambda}}_{m} \in \mathbb{C}^{M \times M}$ is formed by taking the first $M$ columns and rows of $\boldsymbol{\Lambda}_{m}$. The equality in (40) is due to the fact that $\overline{\boldsymbol{\Sigma}}_{m}, \overline{\boldsymbol{\Sigma}}_{m-1}$, and $\overline{\boldsymbol{\Lambda}}_{m}$ have the same distribution in (2) and $E\left[\operatorname{tr}\left(\overline{\boldsymbol{\Sigma}}_{m}^{2}\right)\right]=E\left[\operatorname{tr}\left(\overline{\boldsymbol{\Sigma}}_{m-1}^{2}\right)\right]=E\left[\operatorname{tr}\left(\overline{\boldsymbol{\Lambda}}_{m}^{2}\right)\right]$. With (2), the second term $E\left[\left\|\mathbf{H}_{m} \mathbf{F}_{m-1}\right\|_{F}^{2}\right]$ on the 1.h.s. of (11) is lower bounded by

$$
\begin{aligned}
E\left[\left\|\mathbf{H}_{m} \mathbf{F}_{m-1}\right\|_{F}^{2}\right]= & E\left[\epsilon^{2}\left\|\mathbf{H}_{m-1} \mathbf{F}_{m-1}\right\|_{F}^{2}+\left(1-\epsilon^{2}\right)\left\|\mathbf{N}_{m} \mathbf{F}_{m-1}\right\|_{F}^{2}\right. \\
& \left.+2 \epsilon \sqrt{1-\epsilon^{2}} \operatorname{Re}\left(\operatorname{tr}\left(\mathbf{F}_{m-1}^{*} \mathbf{H}_{m-1}^{*} \mathbf{N}_{m} \mathbf{F}_{m-1}\right)\right)\right] \\
\geq \epsilon^{2} E\left[\left\|\overline{\boldsymbol{\Sigma}}_{m-1} \overline{\mathbf{V}}_{m-1}^{*} \mathbf{F}_{m-1}\right\|_{F}^{2}\right] & \\
& \quad+\left(1-\epsilon^{2}\right) E\left[\left\|\overline{\mathbf{\Lambda}}_{m} \overline{\mathbf{P}}_{m}^{*} \mathbf{F}_{m-1}\right\|_{F}^{2}\right] .(41)
\end{aligned}
$$

In (41) we use $E\left[\operatorname{Re}\left(\operatorname{tr}\left(\mathbf{F}_{m-1}^{*} \mathbf{H}_{m-1}^{*} \mathbf{N}_{m} \mathbf{F}_{m-1}\right)\right)\right]=0$, where $E\left[\mathbf{H}_{m-1}^{*} \mathbf{N}_{m}\right]=\mathbf{0}_{M_{t} \times M_{t}}$. From (40) and (41), we have an upper bound as shown at the top of this page. This bound follows from the facts $\operatorname{tr}(\mathbf{A B}) \leq \operatorname{tr}(\mathbf{A}) \operatorname{tr}(\mathbf{B})$. Now, plugging the definition of the chordal distance yields (11). Note that when $M_{r}=M=1$, (11) becomes an equality because in this case (41) holds as an equality and for the scalar values $\operatorname{tr}(a b)=a b$.

\section{B. Proof of Lemma 1}

We prove Lemma 1 by dividing cases for $m=0$ and $m \geq 1$. 1) For $m=0$ : Consider the quantization error $q_{0}=E\left[\min _{\mathbf{F} \in \mathcal{F}_{0}} d_{c}^{2}\left(\mathbf{F}, \overline{\mathbf{V}}_{0}\right)\right]$. The isotropically distributed source $\overline{\mathbf{V}}_{0}$ is quantized by $B$ bits random matrix codebook $\mathcal{F}_{0}$. The complementary cumulative distribution function (CCDF) of the random variable $\min _{\mathbf{F} \in \mathcal{F}_{0}} d_{c}^{2}\left(\mathbf{F}, \overline{\mathbf{V}}_{0}\right)$ is given by $\left(1-\Upsilon_{0}(x)\right)^{K}$, where $\Upsilon_{0}(x)=\operatorname{Pr}\left(\overline{\mathbf{V}}_{0}: d_{c}^{2}\left(\mathbf{F}, \overline{\mathbf{V}}_{0}\right) \leq x\right)$. Note that when $r \leq 1$, the volume of a spherical cap $\mathcal{S}_{\mathbf{A}}(r)$ in (14) is found in a closed-form [38]

$$
\operatorname{Pr}\left(\mathcal{S}_{\mathbf{A}}(r)\right)=C_{M_{t}, M}\left(r^{2}\right)^{M\left(M_{t}-M\right)}
$$

where $\quad C_{M_{t}, M}=\left(\Gamma\left(M\left(M_{t}-M\right)+1\right)\right)^{-1} \prod_{i=1}^{M} \frac{\Gamma\left(M_{t}-i+1\right)}{\Gamma(M-i+1)} \quad$ and $0<C_{M_{t}, M} \leq 1$. We denote $\kappa=M\left(M_{t}-M\right)$. Note that from (42), we have $\Upsilon_{0}(x)=C_{M_{t}, M} x^{\kappa}$ for $x \leq 1$. Then,

$$
\begin{aligned}
q_{0} & =E\left[\min _{\mathbf{F} \in \mathcal{F}_{0}} d_{c}^{2}\left(\mathbf{F}, \overline{\mathbf{V}}_{0}\right)\right] \\
& =\int_{0}^{1}\left(1-C_{M_{t}, M} x^{\kappa}\right)^{K} d x+\int_{1}^{M}\left(1-\Upsilon_{0}(x)\right)^{K} d x \\
& =\frac{1 / \kappa}{\left(C_{\left.M_{t}, M\right)^{\frac{1}{\kappa}}}\right.} \int_{0}^{C_{M_{t}, M}} z^{\frac{1}{\kappa}-1}(1-z)^{K} d z+\int_{1}^{M}\left(1-\Upsilon_{0}(x)\right)^{K} d x \\
& \leq \underbrace{\frac{1 / \kappa}{\left(C_{M_{t}, M}\right)^{\frac{1}{\kappa}}} \beta\left(C_{M_{t}, M} ; \frac{1}{\kappa}, K+1\right)}_{\triangleq D_{0}}+M\left(1-C_{\left.M_{t}, M\right)^{K}}\right.
\end{aligned}
$$

The result in (43) is obtained by changing variable $C_{M_{t}, M} x^{\kappa}=$ $z$. The first term in (44) follows from the definition of the incomplete beta function ${ }^{3}$. For future reference, we denote the first term in (44) as $D_{0}$.

Now, we investigate the limiting behavior of (44) as $B \rightarrow$ $\infty$. First, we consider the upper bound on $D_{0}$ in (44)

$$
D_{0} \leq \frac{1}{\left(C_{M_{t}, M}\right)^{\frac{1}{\kappa}}} \frac{1}{\kappa} \beta\left(\frac{1}{\kappa}, 2^{B}+1\right) \leq\left(C_{M_{t}, M} 2^{B}\right)^{-\frac{1}{\kappa}},
$$

where $\beta(a, b)$ denotes the beta function with parameter $a$ and $b$. The first step follows from the bound $\beta(c ; a, b) \leq \beta(a, b)$ for $c \leq 1$. The second step comes from the equality $\frac{1}{\kappa} \beta\left(\frac{1}{\kappa}, 2^{B}+\right.$ $1)=2^{B} \beta\left(\frac{1}{\kappa}+1,2^{B}\right)$ and the bound $2^{B} \beta\left(\frac{1}{\kappa}+1,2^{B}\right) \leq 2^{-\frac{B}{\kappa}}$ (see Lemma 1 in [39]). Then, examination of the order of convergence of the quantity in (44) follows

$$
\begin{aligned}
\lim _{B \rightarrow \infty} E\left[2^{\frac{B}{\kappa}}\left(\min _{\mathbf{F} \in \mathcal{F}_{0}} d_{c}^{2}\left(\mathbf{F}, \overline{\mathbf{V}}_{0}\right)\right)\right] & \leq \lim _{B \rightarrow \infty} 2^{\frac{B}{\kappa}}\left(D_{0}+M\left(1-C_{M_{t}, M}\right)^{2^{B}}\right) \\
& \leq C_{M_{t}, M}^{-\frac{1}{\kappa}}
\end{aligned}
$$

where the first step is due to (44). The second step is due to the bound in (45) and the fact that as $B$ tends to infinity, $\left(1-C_{M_{t}, M}\right)^{2^{B}}$ dominates $2^{\frac{B}{\kappa}}$ and $\lim _{B \rightarrow \infty}\left(1-C_{M_{t}, M}\right)^{2^{B}} 2^{\frac{B}{\kappa}}=0$. Thus, when $B$ is large enough, we can write (44) as

$$
q_{0} \leq D_{0}+o(1) \text {. }
$$

Note that the bound in (46) holds as an equality if $M\left(1-C_{M_{t}, M}\right)^{2^{B}}=0$, which is achieved from (44) when $M=1$ or $M=M_{t}-1$ (i.e., $C_{M_{t}, M}=1$ ).

Now, plugging (13) and the bound in (46) into (12) yields

$$
v_{1} \leq \epsilon^{2} D_{0}+\left(1-\epsilon^{2}\right) \frac{\kappa}{M_{t}}+o(1) .
$$

The (47) provides an upper bound for the average directional variation from $\mathbf{F}_{0}$ to $\overline{\mathbf{V}}_{1}$ measured by chordal metric, which is asymptotically tight as $B \rightarrow \infty$. By observing the dominate terms in (47) and with the large $B$ assumption, the square of the spherical cap radius $r_{1}^{2}$ of $\mathcal{F}_{1}$ is determined as

$$
r_{1}^{2}=\epsilon^{2} D_{0}+\left(1-\epsilon^{2}\right) \frac{\kappa}{M_{t}} .
$$

Then, the $\mathcal{F}_{1}$ is generated by drawing $2^{B}$ codewords independently from the isotropic distribution in $\mathcal{S}_{\mathbf{F}_{0}}\left(r_{1}\right)$.

Before we proceed, we investigate a relation between $q_{0}$ and the radius of the quantization cell incurred in $\mathcal{F}_{0}$. For any codebook $\mathcal{F}_{0}$, the subadditivity of the probability measure gives

$$
\begin{aligned}
\operatorname{Pr}\left\{\overline{\mathbf{V}}_{0}: \min _{\mathbf{F}_{0, i} \in \mathcal{F}_{0}} d_{c}^{2}\left(\mathbf{F}_{0, i}, \overline{\mathbf{V}}_{0}\right) \leq x\right\} & \leq \sum_{i=1}^{K} \operatorname{Pr}\left(\overline{\mathbf{V}}_{0}: d_{c}^{2}\left(\mathbf{F}_{0, i}, \overline{\mathbf{V}}_{0}\right) \leq x\right) \\
& =K \operatorname{Pr}(\mathcal{S}(\sqrt{x})) .
\end{aligned}
$$

${ }^{3}$ For $\quad c \leq 1$, the incomplete beta function is defined by
$\int_{0}^{c} z^{a-1}(1-z)^{b-1} d z=\beta(c ; a, b)$. 
Here, we omit the subscript in $\mathcal{S}(\sqrt{x})$ because the formula (42) is invariant to choice of the center. The bound in (49) is achieved if the quantization regions of $\mathcal{F}_{0}$ form ideal Voronoi partitions, i.e., each quantization cell has the same radius $\alpha_{0}$, where $\alpha_{0}$ satisfies $K \operatorname{Pr}\left(\mathcal{S}\left(\alpha_{0}\right)\right)=1$. With the necessary condition $\alpha_{0} \leq 1$, plugging (42) in $K \operatorname{Pr}\left(\mathcal{S}\left(\alpha_{0}\right)\right)=1$ yields

$$
\alpha_{0}=\left(C_{M_{t}, M} 2^{B}\right)^{-\frac{1}{2 \kappa}} .
$$

Comparing (50) and (45), we have $D_{0} \leq \alpha_{0}^{2}$. This reveals that $\alpha_{0}^{2}$ obtained by the sphere covering argument statistically dominates the quantization error $D_{0}$. In the following, for $m \geq$ 1 , the sphere covering argument will be applied to bound $q_{m}$.

2) For $m \geq 1$ : There is no simple closed-form formula for the distribution of $\min _{\mathbf{F} \in \mathcal{F}} d_{c}^{2}\left(\mathbf{F}, \overline{\mathbf{V}}_{m}\right)$. Alternatively, an upper bound on $q_{m}$ is found by applying the sphere covering argument to the area of $\mathcal{S}_{\mathbf{F}_{m-1}}\left(r_{m}\right)$. For this purpose, we equate $K \operatorname{Pr}\left(\mathcal{S}\left(\alpha_{m}\right)\right)=\operatorname{Pr}\left(\mathcal{S}_{\mathbf{F}_{m-1}}\left(r_{m}\right)\right)$ and plug (42) in this equality yielding

$$
\alpha_{m}^{2}=r_{m}^{2} 2^{-\frac{B}{\kappa}},
$$

where the $\alpha_{m}$ is the radius of the ideal Voronoi cell in $\mathcal{S}_{\mathbf{F}_{m-1}}\left(r_{m}\right)$. Note that given $\mathbf{F}_{m-1}$ and $\overline{\mathbf{V}}_{m-1}$, random variables $d_{c}^{2}\left(\mathbf{F}, \overline{\mathbf{V}}_{m}\right)$ with the random codeword $\mathbf{F} \in \mathcal{F}_{m} \subset \mathcal{S}_{\mathbf{F}_{m-1}}\left(r_{m}\right)$ are i.i.d. Thus, the conditional CCDF of $\min _{\mathbf{F} \in \mathcal{F}_{m}} d_{c}^{2}\left(\mathbf{F}, \overline{\mathbf{V}}_{m}\right)$ is given by $\left(1-\varphi_{m \mid m-1}(x)\right)^{K}$, where $\varphi_{m \mid m-1}(x)=\operatorname{Pr}\left(\overline{\mathbf{V}}_{m}: d_{c}^{2}\left(\mathbf{F}, \overline{\mathbf{V}}_{m}\right) \leq x \mid \mathbf{F}_{m-1}, \overline{\mathbf{V}}_{m-1}\right)$ is unknown.

With $\alpha_{m}^{2}$ in (51), the conditional expectation $q_{m \mid m-1} \triangleq$ $E_{\mathcal{F}_{m}, \overline{\mathbf{V}}_{m}}\left[\min _{\mathbf{F} \in \mathcal{F}_{m}} d_{c}^{2}\left(\mathbf{F}, \overline{\mathbf{V}}_{m}\right) \mid \mathbf{F}_{m-1}, \overline{\mathbf{V}}_{m-1}\right]$ is rewritten and bounded by

$$
\begin{aligned}
q_{m \mid m-1} & =\int_{0}^{\alpha_{m}^{2}}\left(1-\varphi_{m \mid m-1}(x)\right)^{2^{B}} d x+\int_{\alpha_{m}^{2}}^{M}\left(1-\varphi_{m \mid m-1}(x)\right)^{2^{B}} d x \\
& \leq \alpha_{m}^{2}+\int_{\alpha_{m}^{2}}^{M}\left(1-\varphi_{m \mid m-1}(x)\right)^{2^{B}} d x
\end{aligned}
$$

We characterize the limiting behavior of $q_{m}$ as $B \rightarrow \infty{ }^{4}$. For fixed $M_{t}$ and $M$,

$$
\begin{aligned}
2^{\frac{B}{\kappa}} q_{m} \stackrel{B}{ } & \leq r_{m}^{2}+E_{\mathcal{F}_{m-1}, \overline{\mathbf{V}}_{m-1}}\left[\lim _{B \rightarrow \infty} \int_{\alpha_{m}^{2}}^{M} 2^{\frac{B}{\kappa}}\left(1-\varphi_{m \mid m-1}(x)\right)^{2^{B}} d x\right] \\
& =r_{m}^{2}
\end{aligned}
$$

where the inequality in (53) follows from the bound in (52) and the equality $\alpha_{m}^{2} 2^{\frac{B}{\kappa}}=r_{m}^{2}$ in (51). The result in (54) follows from the fact that

$$
\lim _{B \rightarrow \infty} \int_{\alpha_{m}^{2}}^{M} 2^{\frac{B}{\kappa}}\left(1-\varphi_{m \mid m-1}(x)\right)^{2^{B}} d x=0
$$

where (55) is obtained by the dominated convergence theorem ${ }^{5}$

\footnotetext{
${ }^{4}$ Note that here, $B \rightarrow \infty$ applies only for the codebook $\mathcal{F}_{m}$, i.e., $\left|\mathcal{F}_{m}\right| \rightarrow \infty$. The sizes of previous codebooks $\mathcal{F}_{m 1}, \ldots, \mathcal{F}_{0}$ does not change.

${ }^{5}$ The theorem is stated as follows: for a sequence of measurable function $f_{n}: x \rightarrow[0, \infty]$, if $\lim _{n \rightarrow \infty} f_{n}(x)=f(x)$ and there exists a dominant function $g(x)$ such that $f_{n}(x) \leq g(x)$ for all $n$ satisfying $\int_{X} g(x) d x<\infty$, then $\lim _{n \rightarrow \infty} \int_{X} f_{n}(x) d x=\int_{X} f(x) d x$.
}

[46]. To provide details, we rewrite the integral in (55) as

$$
\begin{aligned}
\int_{r_{m}^{2} 2^{-\frac{B}{\kappa}}}^{M} 2^{\frac{B}{\kappa}}\left(1-\varphi_{m \mid m-1}(x)\right)^{2^{B}} d x=\int_{r_{m}^{2} 2^{\frac{(a-1) B}{\kappa}}}^{M 2^{\frac{a B}{\kappa}}} \frac{\left(1-\varphi_{m \mid m-1}\left(t 2^{-\frac{a B}{\kappa}}\right)\right)^{2^{B}}}{2^{\frac{(a-1) B}{\kappa}}} d t \\
=\int_{0}^{\infty} \underbrace{\frac{\left(1-\varphi_{m \mid m-1}\left(t 2^{-\frac{a B}{\kappa}}\right)\right)^{2^{B}}}{2^{\frac{(a-1) B}{\kappa}}} \chi_{\left.r_{m}^{2} \frac{(a-1) B}{\kappa}^{\frac{(a)}{\kappa}}, M 2^{\frac{a B}{\kappa}}\right]}(t)}_{\triangleq f_{B}(t)} d t(56)
\end{aligned}
$$

where the first equality follows from a change of variable $2^{\frac{a B}{\kappa}} x=t$ for $a>1$ and the function $\chi_{\mathcal{A}}(t)$ in (56) denotes the indicator function: $\chi_{\mathcal{A}}(t)=1$ if $t \in \mathcal{A}$, and $\chi_{\mathcal{A}}(t)=0$ if $t \notin \mathcal{A}$. To apply the dominated convergence theorem, we need to check two conditions: the existence of $\lim _{B \rightarrow \infty} f_{B}(t)$ and the existence of the dominant function $g(t)$ such that $f_{B}(t) \leq g(t)$ for all $B$ and $\int_{0}^{\infty} g(t) d t<\infty$. From (56), it is straightforward that $\lim _{B \rightarrow \infty} f_{B}(t)=0$. The second condition can be checked by bounding $f_{B}(t) \leq\left(1-\varphi_{m \mid m-1}(t)\right) \chi_{[0, M]}(t)$ and checking that $\int_{0}^{\infty}\left(1-\varphi_{m \mid m-1}(t)\right) \chi_{[0, M]}(t) d t<\infty$. Now, applying the dominated convergence theorem gives

$\lim _{B \rightarrow \infty} \int_{r_{m}^{2} 2^{-\frac{B}{\kappa}}}^{M} 2^{\frac{B}{\kappa}}\left(1-\varphi_{m \mid m-1}(t)\right)^{2^{B}} d x=\int_{0}^{\infty} \lim _{B \rightarrow \infty} f_{B}(t) d t=0$.

Thus, from (54), when the size of $\mathcal{F}_{m}$ is large enough, we can write the bounds on $q_{m}$ and $v_{m+1}$

$$
\begin{aligned}
q_{m} & \leq r_{m}^{2} 2^{-\frac{B}{\kappa}}+o(1) \\
v_{m+1} & \leq \epsilon^{2} r_{m}^{2} 2^{-\frac{B}{\kappa}}+\left(1-\epsilon^{2}\right) \frac{\kappa}{M}+o(1)
\end{aligned}
$$

and $r_{m+1}^{2}$ is obtained by taking dominant term in (58)

$$
r_{m+1}^{2}=\epsilon^{2} r_{m}^{2} 2^{-\frac{B}{\kappa}}+\left(1-\epsilon^{2}\right) \frac{\kappa}{M} .
$$

For $m=1$, with $r_{1}^{2}$ in (48), substituting $r_{1}^{2}$ in (57) and (58) gives bounds for $q_{1}$ and $v_{2}$, where $r_{2}^{2}$ is decided by taking the dominant term of the bound of $v_{2}$. Then, for $m=2,3, \ldots$, recursively applying (57), (58), and (59) gives the general expression for $q_{m}, v_{m+1}$, and $r_{m+1}^{2}$ in (15), (16), and (17), respectively.

\section{Proof of Distance Metric}

Let us define $d\left(\mathbf{U}_{1}, \mathbf{U}_{2}\right) \triangleq \sqrt{1-\frac{1}{M_{t}}\left|\operatorname{tr}\left(\mathbf{U}_{1}^{*} \mathbf{U}_{2}\right)\right|}$. In order to show $d\left(\mathbf{U}_{1}, \mathbf{U}_{2}\right)$ is a metric, we have to prove the following three axioms [47]: (a) $d\left(\mathbf{U}_{1}, \mathbf{U}_{2}\right) \geq 0$ and $d\left(\mathbf{U}_{1}, \mathbf{U}_{2}\right)=0$ if and only if $\mathbf{U}_{1}=\mathbf{U}_{2}$, (b) $d\left(\mathbf{U}_{1}, \mathbf{U}_{2}\right)=d\left(\mathbf{U}_{2}, \mathbf{U}_{1}\right)$, (c) $d\left(\mathbf{U}_{1}, \mathbf{U}_{3}\right) \leq d\left(\mathbf{U}_{2}, \mathbf{U}_{1}\right)+d\left(\mathbf{U}_{2}, \mathbf{U}_{3}\right)$, where $\mathbf{U}_{i} \in \mathcal{U}\left(M_{t}, M_{t}\right)$ for $i \in\{1,2,3\}$. Axiom (a) and axiom (b) are obvious. In order to verify axiom (c), we first provide a lemma that establishes the triangular inequality with vector operands.

Lemma 2: For any unit norm vectors $\mathbf{u}_{i} \in \mathcal{U}\left(M_{t}, 1\right)$ for $i \in\{1,2,3\}$,

$$
\sqrt{1-\left|\mathbf{u}_{1}^{*} \mathbf{u}_{3}\right|} \leq \sqrt{1-\left|\mathbf{u}_{1}^{*} \mathbf{u}_{2}\right|}+\sqrt{1-\left|\mathbf{u}_{2}^{*} \mathbf{u}_{3}\right|} .
$$

Proof: We start from the equality [48] that

$$
\sqrt{1-\left|\mathbf{u}_{1}^{*} \mathbf{u}_{3}\right|}=\min _{\theta} \frac{1}{\sqrt{2}}\left\|\mathbf{u}_{1} e^{j \theta}-\mathbf{u}_{3}\right\|_{2},
$$


where the optimal $\theta$ is the one making $\mathbf{u}_{3}^{*} \mathbf{u}_{1} e^{j \theta}=\left|\mathbf{u}_{3}^{*} \mathbf{u}_{1}\right|$. Then, (61) is equivalently rewritten and bounded by

$$
\begin{aligned}
\sqrt{1-\left|\mathbf{u}_{1}^{*} \mathbf{u}_{3}\right|} & =\min _{\theta, \theta_{2}} \frac{1}{\sqrt{2}}\left\|\mathbf{u}_{1} e^{j \theta}-\mathbf{u}_{2} e^{j \theta_{2}}+\mathbf{u}_{2} e^{j \theta_{2}}-\mathbf{u}_{3}\right\|_{2} \\
& \leq \min _{\theta, \theta_{2}} \frac{1}{\sqrt{2}}\left(\left\|\mathbf{u}_{1} e^{j \theta}-\mathbf{u}_{2} e^{j \theta_{2}}\right\|_{2}+\left\|\mathbf{u}_{2} e^{j \theta_{2}}-\mathbf{u}_{3}\right\|_{2}\right),
\end{aligned}
$$

where in (62), triangular inequality of vector two-norm is used. Let $\theta_{1}=\theta-\theta_{2}$. Then, (62) yields

$$
\begin{aligned}
\sqrt{1-\left|\mathbf{u}_{1}^{*} \mathbf{u}_{3}\right|} & \leq \min _{\theta_{1}} \frac{1}{\sqrt{2}}\left\|\mathbf{u}_{1} e^{j \theta_{1}}-\mathbf{u}_{2}\right\|_{2}+\min _{\theta_{2}} \frac{1}{\sqrt{2}}\left\|\mathbf{u}_{2} e^{j \theta_{2}}-\mathbf{u}_{3}\right\|_{2} \\
& =\sqrt{1-\left|\mathbf{u}_{1}^{*} \mathbf{u}_{2}\right|}+\sqrt{1-\left|\mathbf{u}_{2}^{*} \mathbf{u}_{3}\right|} .
\end{aligned}
$$

This concludes the proof.

Now we are ready to prove Theorem 1. For any unitary matrix $\mathbf{U} \in \mathcal{U}\left(M_{t}, M_{t}\right)$, $\frac{\operatorname{vec}(\mathbf{U})}{\sqrt{M_{t}}}$ forms an $M_{t}^{2}$-dimensional unit norm vector, i.e., $\frac{\operatorname{vec}(\mathbf{U})}{\sqrt{M_{t}}} \in \mathcal{U}\left(M_{t}^{2}, 1\right)$. Then, we can map the matrix trace operation to the vector inner product as

$$
\left|\frac{1}{M_{t}} \operatorname{tr}\left(\mathbf{U}_{1}^{*} \mathbf{U}_{2}\right)\right|=\left|\frac{\operatorname{vec}\left(\mathbf{U}_{1}\right)^{*}}{\sqrt{M_{t}}} \frac{\operatorname{vec}\left(\mathbf{U}_{2}\right)}{\sqrt{M_{t}}}\right| .
$$

Then, from Lemma 2, we obtain the triangular inequality.

\section{Proof of Theorem 2}

For $1 \leq M \leq \frac{M_{t}}{2}$, consider a set of rotation matrices $\mathcal{O}=\left\{\mathbf{O}: \mathbf{O F}_{m-1} \perp \mathbf{F}_{m-1}, \mathbf{O} \in \mathcal{U}\left(M_{t}, M_{t}\right)\right\}$. Any $\widetilde{\mathbf{\Theta}} \in \mathcal{O}$ satisfies $d_{c}^{2}\left(\widetilde{\boldsymbol{\Theta}} \mathbf{F}_{m-1}, \mathbf{F}_{m-1}\right)=M$. Then, given $\widetilde{\boldsymbol{\Theta}} \in \mathcal{O}$, the projected point of $\widetilde{\mathbf{\Psi}}_{\bar{r}_{m}}=w_{m} \mathbf{F}_{m-1}+\bar{r}_{m} \widetilde{\mathbf{\Theta}} \mathbf{F}_{m-1}$, i.e., $\operatorname{proj}\left(\widetilde{\mathbf{\Psi}}_{\bar{r}_{m}}\right)$ produces the farthest point from $\mathbf{F}_{m-1}$ in $\mathcal{U}\left(M_{t}, M\right)$ because the direction of the perturbation added to $w_{m} \mathbf{F}_{m-1}$ in $\widetilde{\mathbf{\Psi}}_{\bar{r}_{m}}$ is orthogonal to $\mathbf{F}_{m-1}$. If we find the $w_{m}$ such that $d_{c}^{2}\left(\operatorname{proj}\left(\widetilde{\boldsymbol{\Psi}}_{\bar{r}_{m}}\right), \mathbf{F}_{m-1}\right)=r_{m}^{2}$, then for any point in $\left\{\boldsymbol{\Psi}_{\bar{r}_{m}}=w_{m} \mathbf{F}_{m-1}+\bar{r}_{m} \boldsymbol{\Theta} \mathbf{F}_{m-1}: \boldsymbol{\Theta} \in \mathcal{U}\left(M_{t}, M_{t}\right)\right\}$, we have $d_{c}^{2}\left(\operatorname{proj}\left(\mathbf{\Psi}_{\bar{r}_{m}}\right), \mathbf{F}_{m-1}\right) \leq r_{m}^{2}$. Note that when $\frac{M_{t}}{2}<M \leq$ $M_{t}-1$, we only need to consider the rotation of the orthogonal complement of $\mathbf{F}_{m-1}$ and it is handled similarly to the case $1 \leq M \leq \frac{M_{t}}{2}$. We omit the case $\frac{M_{t}}{2}<M \leq M_{t}-1$ and focus on $1 \leq M \leq \frac{M_{t}}{2}$.

In order to extract the column subspace of $\widetilde{\mathbf{\Psi}}_{\bar{r}_{m}}=w_{m} \mathbf{F}_{m-1}+\bar{r}_{m} \widetilde{\boldsymbol{\Theta}} \mathbf{F}_{m-1}$, consider $\widetilde{\boldsymbol{\Psi}}_{\bar{r}_{m}}^{*} \widetilde{\boldsymbol{\Psi}}_{\bar{r}_{m}}$ resulting in $\widetilde{\boldsymbol{\Psi}}_{\bar{r}_{m}}^{*} \widetilde{\boldsymbol{\Psi}}_{\bar{r}_{m}}=\left(w_{m}^{2}+\bar{r}_{m}^{2}\right) \mathbf{I}_{M}$ where the compact singular value decomposition of $\widetilde{\boldsymbol{\Psi}}_{\bar{r}_{m}}$ is given by

$$
\widetilde{\mathbf{\Psi}}_{\bar{r}_{m}}=\frac{1}{\sqrt{w_{m}^{2}+\bar{r}_{m}^{2}}} \widetilde{\mathbf{\Psi}}_{\bar{r}_{m}}\left(\sqrt{w_{m}^{2}+\bar{r}_{m}^{2}} \mathbf{I}_{M}\right) \mathbf{I}_{M} .
$$

Then we have $\operatorname{proj}\left(\widetilde{\mathbf{\Psi}}_{\bar{r}_{m}}\right)=\frac{1}{\sqrt{w_{m}^{2}+\bar{r}_{m}^{2}}} \widetilde{\boldsymbol{\Psi}}_{\bar{r}_{m}}$. Now, we decide $w_{m}$ such that $d_{c}^{2}\left(\frac{1}{\sqrt{w_{m}^{2}+\bar{r}_{m}^{2}}} \widetilde{\mathbf{\Psi}}_{\bar{r}_{m}}, \mathbf{F}_{m-1}\right)=r_{m}^{2}$. Solving this equality gives $\bar{r}_{m}^{2}=\frac{\bar{r}_{m}^{2}}{w_{m}^{2}+\bar{r}_{m}^{2}}$, which leads to $w_{m}=\sqrt{1-\bar{r}_{m}^{2}}$.

\section{REFERENCES}

[1] D. J. Love, R. W. Heath Jr., V. K. N. Lau, D. Gesbert, B. D. Rao, and M. Andrews, "An overview of limited feedback wireless communication systems," IEEE Jour. Select. Areas in Commun., vol. 26(8), pp. 13411365, Oct. 2008.
[2] D. J. Love and R. W. Heath Jr., "Limited feedback unitary precoding for spatial multiplexing system," IEEE Trans. Info. Th., vol. 51, no. 8, pp. 2967-1976, Aug. 2005.

[3] W. Santipach and M. L. Honig, "Capacity of a multiple-antenna fading channel with a quantized precoding matrix," IEEE Trans. Info. Th., vol. 55, no. 3, pp. 1218-1234, Mar. 2009.

[4] J. C. Rho and B. D. Rao, "Design and analysis of MIMO spatial multiplexing systems with quantized feedback," IEEE Trans. Sig. Proc., vol. 54, no. 8, pp. 4730-4740, Dec. 2006.

[5] R. H. Etkin and D. N. C. Tse, "Degree of freedom in some underspread MIMO fading channel," IEEE Trans. Info. Th., vol. 52, no. 4, pp. 15761608, Apr. 2006.

[6] A. Gersho and R. M. Gray, Vector Quantization and Signal Compression. Boston, MA: Springer, 1992.

[7] B. Banister and J. Zeidler, "Feedback assisted transmission subspace tracking for MIMO systems," IEEE Jour. Select. Areas in Commun., vol. 21, no. 3, pp. 452-463, Apr. 2003.

[8] J. Yang and D. Williams, "Transmission subspace tracking for MIMO systems with low-rate feedback," IEEE Trans. Commun., vol. 55, no. 8 , pp. 1629-1639, Aug. 2007.

[9] B. Mondal and R. W. Heath, "Channel adaptive quantization for limited feedback MIMO beamforming system," IEEE Trans. Sig. Proc., vol. 54, no. 12, pp. 4717-4729, Dec. 2006.

[10] R. Samanta and R. W. Heath, "Codebook adaptation for quantized MIMO beamforming systems," in Proc. 39th Asilomar conference, Oct. 2005, pp. 376-380.

[11] R. W. Heath Jr., T. Wu, and A. C. K. Soong, "Progressive refinement of beamforming vectors for high-resolution limited feedback," EURASIP Jour. Advances in Sig. Proc., vol. Article ID 463823, 13 pages, 2009.

[12] C. Simon and G. Leus, "Feedback quantization for linear precoded spatial multiplexing," EURASIP Jour. Advances in Sig. Proc., vol. 2008: Article ID 683030, 13 pages, 2008.

[13] G. Leus and C. Simon, "Quantized feedback and feedback reduction for precoded spatial multiplexing MIMO systmes," in Proc. of the Int. Symp. on Sig. Proc. and its Appl. 2007 (ISSPA 2007), Feb. 2007.

[14] T. Kim, B. Clerckx, D. J. Love, and S. J. Kim, "Differential rotation feedback MIMO system for temporally correlated channel," in Proc. IEEE Globecom, Dec. 2008.

[15] J.-L. Pan, R. Olesen, D. Grieco, and C.-P. Yen, "Efficient feedback design for MIMO SC-FDMA systems," in Proc. Veh. Tech. Conf. 2007Spring, IEEE 65th, Apr. 2007, pp. 2399-2403.

[16] K. Huang, R. W. Heath, and J. G. Andrew, "Limited feedback beamforming over temporally-correlated channels," IEEE Trans. Sig. Proc., vol. 57 , no. 5, pp. 1-18, May 2009.

[17] T. Kim, D. J. Love, and B. Clerckx, "Leveraging temporal correlation for limited feedback multiple antennas systems," in Proc. IEEE Int. Conf. on Acoustics, Speech and Sig. Proc., Mar. 2010.

[18] H.-L. Maattanen, O. Tirkkonen, and T. Roman, "Defferential CQI optimization for MIMO with codebook based precoding," in IEEE Sig. Proc. Advances in Wireless Commun., Jun 2009.

[19] E. Biglieri, R. Calderbank, A. Constantinides, A. Goldsmith, A. Paulraj, and H. V. Poor, MIMO Wireless Communications. Cambridge Univ. Press, 2007.

[20] J. C. Rho and B. D. Rao, "Multiple antenna channels with partial channel state information at the transmitter," IEEE Trans. Wireless Commun., vol. 3, no. 2, pp. 677-688, May 2004.

[21] R. S. Blum, "MIMO with limited feddback of channel state information," in Proc. IEEE Int. Conf. on Acoustics, Speech and Sig. Proc., vol. 4, 2003, pp. 89-92.

[22] V. Lau, Y. Liu, and T. Chen, "On the design of MIMO block-fading channels with feedback-link capacity constraint," vol. 52, no. 1, pp. $62-$ 70, Jan. 2004.

[23] "IEEE P802.16m-2008 draft standard for local and metropollitan area network," in IEEE Standard 802.16m, 2008.

[24] "3GPP LTE Release-8 V0.0.9: Evolved Packet System RAN Part," in $3 G P P$ LTE, Dec. 2009.

[25] D. Sacristan and A. Pascual-Iserte, "Differential feedback of MIMO channel correlation matrices based on geodesic curves," in Proc. IEEE Int. Conf. on Acoustics, Speech and Sig. Proc., Apr. 2009.

[26] D. Sacristan, F. Kaltenberger, A. Pascual-Iserte, and A. Perez, "Differential feedback in MIMO communications: performance with delay and real channel measurments," in Proc. Int. ITG Workshop on Smart Antennas (WSA 2009), Feb. 2009.

[27] M. Talih, "Geodesic Markov chains on covariance matrices," SAMSI Technical Report No. 2007-4, pp. 376-380, Mar. 2007. 
[28] S. C. Douglas, S. Amari, and S. Y. Kung, "On gradient adaptation with unit-norm constrations," IEEE Trans. Sig. Proc., vol. 48, no. 6, pp. 18431847, June 2000.

[29] J. H. Manton, "Optimization algorithms exploiting unitray constraints," IEEE Trans. Sig. Proc., vol. 50, no. 3, pp. 635-650, Mar. 2002.

[30] J.-F. Yang and M. Kaveh, "Adaptive eigenspace algorithms for direction or frequency estimation and tracking," IEEE Trans. on Acoustics, Speech and Sig. Proc., vol. 36, no. 2, pp. 241-251, Feb. 1988.

[31] G. S. Watson, Statistics on spheres. Wiley, New York, 1983.

[32] J. Choi and J. R. W. Heath, "Interpolation based transmit beamforming for MIMO-OFDM with limited feedback," IEEE Trans. Sig. Proc., vol. 53, no. 11, pp. 1425-1435, Nov. 2005.

[33] J. Choi, B. Mondal, and J. R. W. Heath, "Interpolation based unitary precoding for spatial multiplexing MIMO-OFDM with limited feedback," IEEE Trans. Sig. Proc., vol. 54, no. 12, pp. 4730-4740, Dec. 2006

[34] J. G. Proakis, Digital Communications, 4th ed. Mc Graw Hill, 2000.

[35] W. Dai, Y. Liu, V. K. N. Lau, and B. Rider, "On the information rate of MIMO systemas with finite rate channel state feedback and power on/off strategy," in Proc. IEEE Int. Symp. Info. Th., Sept. 2005.

[36] D. J. Love and R. W. Heath Jr., "Multimode precoding for MIMO wireless system," IEEE Trans. Sig. Proc., vol. 53, no. 10, pp. 3674 3687, Oct. 2005.

[37] A. Edelman, T. A. Arias, and S. T. Smith, "The geometry of algorithms with orthogonality constraints,' SIAM J. Matrix Anal. Appl., vol. 20, no. 2, pp. 303-353, Oct. 1998.

[38] W. Dai, Y. Liu, and B. Rider, "Quantization bound on Grassmann manifolds and applications ot mimo communications," IEEE Trans. Info. Th., vol. 54, no. 3, pp. 1108-1123, Mar. 2008.

[39] N. Jindal, "MIMO broadcast channels with finite-rate feedback," IEEE Trans. Info. Th., vol. 52, no. 11, pp. 5045-5060, Nov. 2006.

[40] S. Gallot, D. Hulin, and J. Lafontaine, Riemannian Geometry, 3rd ed. New York, NY: Springer, 2004.

[41] S. Roman, Advanced Linear Algebra, 2nd ed. Springer, 2005.

[42] I. H. Kim, S. Y. Park, D. J. Love, and S. J. Kim, "Improved multiuser MIMO unitary precoding using partial channel state information and insight from the reimannian manifold," IEEE Trans. Wireless Commun., vol. 8, no. 8, pp. 4014-4023, Aug. 2009.

[43] D. J. Love and R. W. Heath Jr., "Limited feedback diversity techniques for correlated channels," IEEE Trans. Veh. Tech., vol. 55, no. 2, pp. 713-722, Mar. 2006.

[44] G. H. Golub and C. F. V. Loan, Matrix Computation, 3rd ed. Baltimore and London: Johns Hopkins Univ. Press, 1996.

[45] J. Salo, G. Del Galdo, J. Salmi, P. Kyosti, M. Milojevic, D. Laselva, and C. Schneider. (2005, Jan.) MATLAB implementation of the 3GPP Spatial Channel Model (3GPP TR 25.996). [Online]. Available: http://www.tkk.fi/Units/Radio/scm/

[46] P. Billingsley, Probability and Measure, 3rd ed. New York: Wiley Interscience, 1995.

[47] H. L. Royden, Real Analysis, 3rd ed. New Jersey, NJ: Prentice Hall, 1988.

[48] D. J. Love and R. W. Heath Jr., "Equal gain transmission in multipleinput multiple-output wireless systems," IEEE Trans. Commun., vol. 51, no. 7, pp. 1102-1110, 2003.

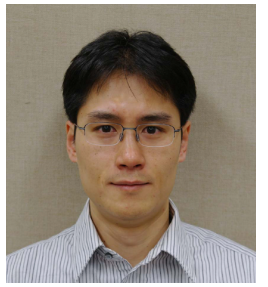

Taejoon Kim (S'08) received the B.S. degree (with highest honors) in electrical engineering from Sogang University, Seoul, Korea, in 2002, and M.S. degree in electrical engineering from the Korea Advanced Institute of Science and Technology (KAIST), Daejeon, Korea, in 2004. From 2004 to 2006, he was with Electronics and Telecommunications Research Institute (ETRI), Daejeon, Korea. Since 2007, he has worked towards the Ph.D. degree at Purdue University. He was a Summer Intern in the Samsung R\&D Center, Richardson, TX, in 2008 and in DSPS R\&D Center, Texas Instrument, Dallas, TX, in 2010, respectively.

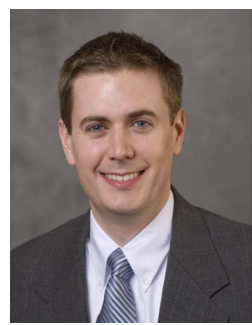

David J. Love (S'98 - M'05 - SM'09) received the B.S. (with highest honors), M.S.E., and Ph.D. degrees in electrical engineering from the University of Texas at Austin, in 2000, 2002, and 2004, respectively. Since August 2004, he has been with the School of Electrical and Computer Engineering, Purdue University, West Lafayette, IN, where he is now an Associate Professor. Dr. Love has served as a Guest Editor for the IEEE Journal on Selected Areas in Communications and serves as an Associate Editor for the IEEE Transactions on Communications. Dr. Love is a member of Tau Beta Pi and Eta Kappa Nu. In 2003, he received the IEEE Vehicular Technology Society Daniel Noble Fellowship. He received the 2009 IEEE Transactions on Vehicular Technology Jack Neubauer Memorial Award for the best systems paper and the 2010 Purdue Eta Kappa $\mathrm{Nu}$ Outstanding Teacher Award. His research interests are in the design and analysis of communication systems.

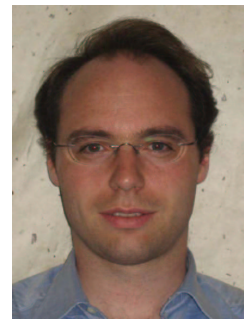

Bruno Clerckx received the M.S. and Ph.D. degree in applied science from the Universite Catholique de Louvain, Belgium. He held visiting research positions at Stanford University, USA, and Eurecom Institute, France. He is currently with Samsung Advanced Institute of Technology, Samsung Electronics, Korea. He is the author or coauthor of one book on MIMO wireless communications and about 50 research papers. He has been actively contributing to 3GPP LTE/LTE-Advanced and IEEE $802.16 \mathrm{~m}$ since 2007. 